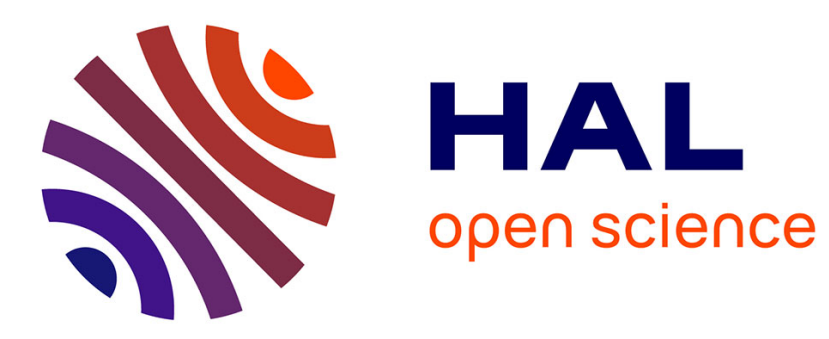

\title{
Real-time non-rigid target tracking for ultrasound-guided clinical interventions
}

Cornel Zachiu, Mario Ries, Pascal Ramaekers, Jean-Luc Guey, Chrit T. W. Moonen, Baudouin Denis de Senneville

\section{- To cite this version:}

Cornel Zachiu, Mario Ries, Pascal Ramaekers, Jean-Luc Guey, Chrit T. W. Moonen, et al.. Real-time non-rigid target tracking for ultrasound-guided clinical interventions. Physics in Medicine and Biology, 2017, 62 (20), pp.8154 - 8177. 10.1088/1361-6560/aa8c66 . hal-01617624

\section{HAL Id: hal-01617624 \\ https://hal.science/hal-01617624}

Submitted on 21 Dec 2018

HAL is a multi-disciplinary open access archive for the deposit and dissemination of scientific research documents, whether they are published or not. The documents may come from teaching and research institutions in France or abroad, or from public or private research centers.
L'archive ouverte pluridisciplinaire HAL, est destinée au dépôt et à la diffusion de documents scientifiques de niveau recherche, publiés ou non, émanant des établissements d'enseignement et de recherche français ou étrangers, des laboratoires publics ou privés. 


\title{
Real-Time Non-Rigid Target Tracking for Ultrasound-Guided Clinical Interventions
}

\author{
C Zachiu ${ }^{1}$, M Ries ${ }^{1}$, P Ramaekers ${ }^{1}$, J-L Guey ${ }^{2}$, C T W \\ Moonen $^{1}$ and B Denis de Senneville ${ }^{1,3}$ \\ ${ }^{1}$ Imaging Division, UMC Utrecht, Heidelberglaan 100, 3508 GA, Utrecht, \\ Netherlands \\ ${ }^{2}$ Imasonic SAS, 4 rue des Savourots, 70190 Voray sur l'Ognon, France \\ ${ }^{3}$ Institut de Mathématiques de Bordeaux (IMB), UMR 5251 CNRS/University of \\ Bordeaux, F-33400 Talence, France. \\ E-mail: C.Zachiu@umcutrecht.nl
}

\begin{abstract}
Biological motion is a problem for non- or mini-invasive interventions when conducted in mobile/deformable organs due to the targeted pathology moving/deforming with the organ. This may lead to high miss rates and/or incomplete treatment of the pathology. Therefore, real-time tracking of the target anatomy during the intervention would be beneficial for such applications.

Since the aforementioned interventions are often conducted under B-mode ultrasound (US) guidance, target tracking can be achieved via image registration, by comparing the acquired US images to a separate image established as positional reference. However, such US images are intrinsically altered by speckle noise, introducing incoherent gray-level intensity variations. This may prove problematic for existing intensity-based registration methods. In the current study we address US-based target tracking by employing the recently proposed EVolution registration algorithm. The method is, by construction, robust to transient gray-level intensities. Instead of directly matching image intensities, EVolution aligns similar contrast patterns in the images. Moreover, the displacement is computed by evaluating a matching criterion for image sub-regions rather than on a point-by-point basis, which typically provides more robust motion estimates. However, unlike similar previously published approaches, which assume rigid displacements in the image sub-regions, the EVolution algorithm integrates the matching criterion in a global functional, allowing the estimation of an elastic dense deformation.

The approach was validated for soft tissue tracking under free-breathing conditions on the abdomen of 7 healthy volunteers. Contact echography was performed on all volunteers, while 3 of the volunteers also underwent standoff echography. Each of the two modalities is predominantly specific to a particular type of non- or mini-invasive clinical intervention. The method demonstrated on average an accuracy of $\sim 1.5 \mathrm{~mm}$ and submillimeter precision. This, together with a computational performance of 20 images/s make the proposed method an attractive solution for real-time target tracking during US-guided clinical interventions.
\end{abstract}

Keywords: Deformable image registration, Ultrasound-guidance, Real-time system. Submitted to: Phys. Med. Biol. 


\section{Introduction}

B-mode ultrasound (US) guidance is currently common practice for a large variety of percutaneous clinical applications (Dogra \& Saad 2009). For example, US-guided percutaneous needle biopsies are routinely performed when abnormal tissues are discovered inside a patients body (Dogra \& Saad 2009, Gupta \& Madoff 2007). Collecting samples of the tissue can become, however, problematic when the procedure is conducted in mobile and/or deformable organs, in particular if limited US-visibility of the lesion requires stereotactic guidance. Due to organ motion and/or deformation induced by the needle insertion process, the position of the targeted tissue may change during the intervention. This can prolong the procedure, lead to sub-optimal sampling or to missing the target altogether, increasing the risk of false negatives. With the development of robotic tissue sampling systems, this becomes particularly problematic (Kaye et al. 2014, Liang et al. 2010).

Another example of percutaneous clinical applications that are often conducted in conjunction with B-mode US-guidance are thermal ablations of tumorous tissues via laser, radiofrequency (RF) or high intensity focused ultrasound (HIFU) (Chu \& Dupuy 2014, Mou et al. 2016, Hofer et al. 2008, Zhang \& Wang 2010). HIFU in particular, is currently the only percutaneous thermal ablation procedure that is completely non-invasive (Chu \& Dupuy 2014). It has already been applied successfully for the treatment of numerous pathologies such as uterine fibroids (Chapman \& ter Haar 2007), prostate cancer (Blana et al. 2008), liver tumors (Okada et al. 2006, Li et al. 2007), thyroid nodules (Kovatcheva et al. 2015a), palliation of bone metastases (Liberman et al. 2009), breast fibroadenoma (Kovatcheva et al. 2015b) and tremordominant Parkinson's disease (Zaaroor et al. 2017). Treatment becomes challenging, however, when therapy is conducted in the abdomen and the lower thorax. Physiological motion such as respiration, peristalsis and/or spontaneous motion, may cause the targeted area to continuously change position over the duration of the intervention. If left un-addressed, this can lead to the therapeutic energy dose to be diverted from the target area and in turn to the under-treatment of the pathology. If motion effects are severe, damage to organs at risk may occur (Ries et al. 2010, Auboiroux et al. 2012, Holbrook et al. 2014). A solution to this is to use the acquired B-mode US images to track the motion of the target area, followed by a corresponding adaptation of the HIFU energy delivery. In this sense, previous studies have proposed and developed integrated systems capable of delivering US-based motion compensated HIFU therapies. For example, Seo et al designed a system consisting of two US imaging probes integrated with a HIFU transducer (Seo et al. 2011a, Seo et al. 2011b, Seo et al. 2016). One probe was placed at the center of the parabolic transducer, while the second was placed on the edge of the transducer at a $45^{\circ}$ angle with respect to the first one. This arrangement of the US probes provided two orthogonal US imaging planes intersecting at the transducer focal point, offering the possibility to track both in-plane and out-of-plane motion. The displacements were estimated either by matching the intra-operative target surface to a 
pre-operative model (Seo et al. 2011a, Seo et al. 2011b) or by tracking the discriminative speckle pattern produced in the US images by the HIFU-induced lesion (Seo et al. 2016). Beam steering with respect to the estimated motion was achieved by visual servoing implemented in a three-axis robot. Promising results were obtained with tracking errors of 1.5 - $2 \mathrm{~mm}$, however, in-vivo validation is yet to be performed. A different integrated system capable of providing simultaneous magnetic resonance imaging (MRI) and US guidance during HIFU therapies was developed by Auboiroux et al and is described in several related studies (Auboiroux et al. 2012, Petrusca et al. 2013, Preiswerk et al. 2014). US guidance was provided by a probe placed on a custom-built support, allowing 5 degrees of freedom for movement around the patient. The support was attached to the MR patient table, which also contained the HIFU transducer. US-based motion estimation was achieved both by an optical flow approach (Auboiroux et al. 2012) and also by a more advanced estimation-prediction algorithm relying on a populationbased statistical model (Preiswerk et al. 2014). This lead to motion estimates with a 1-3 mm accuracy, available with near real-time latency.

In summary, in order to facilitate motion compensated therapy deliveries, accurate and precise motion estimation algorithms are paramount. A large variety of US-based motion tracking methods have already been proposed in the literature (De Luca et al. 2015b). The majority of these methods aim to estimate the position of a structure-of-interest at a particular time-instant by aligning an US-image (also called moving image) to a reference image through a process called registration (Mani \& Arivazhagan 2013). According to the classification made in the review work of de Luca et al (De Luca et al. 2015b), registration methods can be coarsely divided into three categories: intensity-based, feature-based and hybrid. Hybrid approaches, in particular, have the benefit of an improved accuracy and precision of the estimated deformation compared to intensity-based and feature-based methods (De Luca et al. 2015b). Cifor et al (Cifor et al. 2013), for example, propose a so-called Hybrid Feature-based Diffeomorphic (HFD) registration, inspired by the diffeomorphic Log-Demons registration framework. The method estimates the deformation between two US images by the means of a diffeomorphic transformation, which in turn is computed based on a set of demonlike forces (Thirion 1998) driven by local image features, including: image intensity, local phase (Woo et al. 2009) and phase congruency (Kovesi 2000). The method was used to track liver tumors on B-mode US images acquired on 7 patients. Results have shown an improvement of the Dice similarity coefficient (Dice 1945) from an average of $73.9 \pm 19.5$, in the absence of tracking, to $90.4 \pm 5.5$. A drawback of the approach is that it requires the optimization and/or computation of several input parameters to which the method is more or less sensitive, encumbering its application on a case-by-case basis. Moreover, the method is computationally demanding, requiring $5 \mathrm{~min}$ for registering one pair of 2D images. Hybrid methods, making use of both image intensity and contour information, have also been proposed in the past for contour tracking in B-mode US images (Li et al. 2005, Wang et al. 2010, Huang et al. 2014). The approach proposed by $\mathrm{Li}$ et $a l$ ( $\mathrm{Li}$ et al. 2005) relies on the active contour framework (Kass et al. 1988) 
in order to track from frame-to-frame the deformation of a manually-selected image contour. As an application scenario, the method was employed for automatic US-based tracking of tongue movements during speech. It was demonstrated to have a good accuracy, with an average sum of distances between the automatically tracked tongue contour and the delineations performed by experts of $0.54-1.06 \mathrm{~mm}$. While robust, the method requires a moderate amount of user interaction. This includes manual initialization of the contour, its re-initialization in case of large inter-frame displacements and the tuning of several input parameters. Moreover, since the method uses the state of the contour from the previous frame as an initialization for the current frame, it is susceptible to error accumulation. The approaches proposed by Wang and Huang (Wang et al. 2010, Huang et al. 2014), on the other hand, make use of prior information learned on training datasets in order to perform automatic contour detection and/or motion characterization. Both methods were used in the context of echocardiography, for automatic tracking of heart contours. The solution proposed by Wang et al was shown to have an accuracy and precision of $2.68 \mathrm{~mm}$ and $2.63 \mathrm{~mm}$, respectively, for tracking "end - systole" - "end - diastole" heart deformations. Similarly, the approach of Huang et al demonstrated promising results, with a Hausdorff distance (Huttenlocher et al. 1993) between automatically-tracked and expert-defined contours of $2.95 \pm 0.62 \mathrm{~mm}$ for the endocardium and $3.03 \pm 0.76 \mathrm{~mm}$ for the epicardium. The method was also evaluated in terms of the Dice similarity coefficient, with an average value after registration of 93 and 97 for the endocardium and epicardium, respectively. Such approaches, however, have complex numerical schemes and require moderate user interaction. Moreover, due to the fact that they are learning-based, the prior information has to be updated on a case-by-case basis, having also difficulties in handling deformation patterns that are not included in the learned atlas/dictionary. As previously mentioned, many other US-based tracking solutions are available in prior art, dedicated to various applications. While individual validation of the methods was performed using several different criteria, it was concluded in the review work of de Luca et al (De Luca et al. 2015b) that, on average, existing US-based tracking methods can achieve an accuracy of $1-2 \mathrm{~mm}$.

For the interventional guidance of mini- or non-invasive procedures, a motion tracking solution with real-time capabilities is of particular importance. In the scope of this work, a registration method is defined as "real-time" if it is capable of aligning two images with a sufficiently low latency such that the monitoring and control of the intervention is not hampered by the associated delay. Naturally, the term "sufficient" depends on the particularities of the application. In this sense the so-called variational registration methods (Weickert et al. 2003) are a particularly attractive option for real-time USbased motion tracking (Ries et al. 2010). This is due to both their fast numerical schemes and the reduced number of required input parameters, with the latter leading to an increased ease-of-use in a clinical setting on a case-by-case basis.

In the present study we propose a real-time US-based motion tracking solution based on the EVolution algorithm, recently proposed by Denis de Senneville et al (Denis de Senneville et al. 2016). EVolution is a variational registration method which, intuitively 
speaking, in order to estimate the deformation between two images, searches for similar local contrast patterns in the two images, which it then tries to align. With respect to the classification made by de Luca et al in (De Luca et al. 2015b), EVolution falls into the category of hybrid registration methods. This is due to the fact that the method computes a deformation field on a point-by-point basis, iteratively minimizing a global matching criterion between the images (which is specific to intensity-based methods), however, it does not operate directly on image intensities, matching instead local contrast patterns in the images (which is historically more used in feature-based approaches). Similar to the methods proposed in (König et al. 2014) and (Kubota et al. 2014), the EVolution algorithm operates on square sub-regions (called patches or windows) rather than on a point-by-point basis. This typically provides a robust local displacement, since the motion in a point-of-interest is calculated based on several pixels/voxels. However, while the aforementioned approaches assume a rigid motion inside the sub-regions, EVolution integrates the patch-based matching criterion in a global variational framework, which allows estimating an elastic deformation on a pointby-point basis.

The current study validates the EVolution algorithm with both ex-vivo and in-vivo USbased motion estimation experiments. The method was tested hereby for both contact echography, which is, for example, specific to needle biopsies, as well as for standoff echography, which is typical for set-ups employed for US-guided HIFU therapies. In particular for the standoff case, the evaluation was conducted directly on a prototype of a clinical US-guided HIFU system developed at our institute, which ultimately imposed additional constraints on obtainable image quality.

\section{Method description}

\subsection{Proposed solution for B-mode US-based motion tracking}

2.1.1. The EVolution algorithm Motion estimation via the EVolution algorithm, implies finding the minimizer of the following functional:

$$
E(T)=\int_{\Omega} e^{-C(T)}+\frac{\alpha}{2}\left(\|\vec{\nabla} u\|^{2}+\|\vec{\nabla} v\|^{2}\right) d \vec{r}
$$

with

$$
C(T)=\frac{\int_{\Gamma}\left|\vec{\nabla}_{I}(T(\vec{r})) \cdot \vec{\nabla}_{J}(\vec{r})\right| d \vec{r}}{\int_{\Gamma}\left\|\vec{\nabla}_{I}(T(\vec{r}))\right\|_{2}\left\|\vec{\nabla}_{J}(\vec{r})\right\|_{2} d \vec{r}}
$$

where $T=(u, v)$ is the $2 \mathrm{D}$ displacement to be estimated, $I$ and $J$ are the reference and the moving image, $\alpha$ is a parameter linking the two terms of the functional, $\vec{\nabla}$ is the spatial gradient operator, $\|\cdot\|_{2}$ is the Euclidean norm, $\Omega$ is the image domain, $\vec{r}$ is a spatial location and $\Gamma$ is a square image patch centered on the pixel of interest. The term $C(T)$ in Eq. 2 can be rewritten as:

$$
C(T)=\frac{\int_{\Gamma} w_{T}(\vec{r})\left|\cos \left(\Delta \theta_{T}(\vec{r})\right)\right| d \vec{r}}{\int_{\Gamma} w_{T}(\vec{r}) d \vec{r}}
$$


with the terms $w_{T}(\vec{r})$ and $\Delta \theta_{T}(\vec{r})$ being computed based on the magnitude $M$ and the phase $\theta$ of the reference and moving image spatial gradients:

$$
\begin{aligned}
w_{T}(\vec{r}) & =M_{I}(T(\vec{r})) M_{J}(\vec{r}) \\
\Delta \theta_{T}(\vec{r}) & =\theta_{I}(T(\vec{r}))-\theta_{J}(\vec{r})
\end{aligned}
$$

The term $C(T)$ can be interpreted as follows: $\left|\cos \left(\Delta \theta_{T}(\vec{r})\right)\right|$ in Eq. 3 favors transformations that align edges in the reference and the moving image, regardless of any possible contrast reversals. This basically makes the algorithm capable of both mono and multi-modal registration. On the other hand, $w_{T}(\vec{r})$ favors strong edges that exist in both images. Thus the method is, by construction, robust to transient structures.

Minimizing $e^{-C(T)}$ alone, however, is a severely ill-posed problem with infinite solutions. For this reason, the functional in Eq. 1 contains a regularization term weighted by $\alpha$, constraining the estimated displacements to be spatially differentiable, which "enforces"/mimics plastic deformations (Horn \& Schunck 1981). This renders the optimization problem overdetermined and therefore solvable with variational methods.

2.1.2. Implemented optimization scheme The EVolution functional was minimized via the iterative procedure described in (Denis de Senneville et al. 2016). Similarly, the iterative process was stopped when the average absolute difference between the displacements at the current and the previous iteration was smaller or equal to $10^{-3}$ pixels.

Due to the strong non-linearity of the functional, the algorithm has difficulties estimating large displacements. For this reason, a coarse-to-fine strategy was adopted in which motion is estimated step-by-step starting from a 16-fold downsampled version of the images up to their original resolution. The displacements estimated at a particular resolution level are then used as an initialization for the next level.

\subsection{Experimental setup}

In the current study, the capabilities of the EVolution algorithm for B-mode US tracking, were validated for both contact and standoff echography, with a separate experiment dedicated to each of the two US-acquisition modalities. The two experiments are detailed in the following sections.

2.2.1. In-vivo contact echography study The current healthy volunteer study was conducted in agreement with the required standards and regulatory requirements. Ethical approval was provided by the Ethics Board of the University Medical Center Utrecht. B-mode US imaging was performed under free-breathing conditions on the liver and one of the kidneys of 7 healthy volunteers. The images were acquired using a Telemed C3.5/60/128Z convex array transducer (number of elements $=128$, radius of curvature $=65 \mathrm{~mm}$, field-of-view $=59^{\circ}$ ) connected to a LS128 EXT-1Z Telemed beamformer. The acquisition sequence employed the following parameters: Frequency 
$=5 \mathrm{MHz}$, Depth $=80 \mathrm{~mm}, \mathrm{FPS}=18$ and pixel size $=0.25 \times 0.25 \mathrm{~mm}^{2}$. The images were saved on disk and processed offline. Since approximately half of the far field-ofview of the images contained either anatomical structures that were not of interest in the scope of this study, either exclusively noise due to signal attenuation, these parts were manually discarded from the images. Note, however, that this step was performed only in the scope of the current work and can be omitted in practice. For computational purposes, the manual discarding of parts of the field-of-view was followed by a spatial sub-sampling of the images by a factor of 2 . These two pre-processing steps resulted in each image in the series having a size of $256 \times 256$ with a pixel size of $0.5 \times 0.5 \mathrm{~mm}^{2}$.

For both the liver and the kidney datasets, three identifiable landmarks were selected, for which the tracking capabilities of the EVolution algorithm was evaluated. More specifically, for the liver this consisted in the lower boundary of segment \# 4 (landmark \# 1) and two branches of the hepatic artery/portal vein (landmarks \# 2 and \# 3). The two blood vessels were chosen in approximately the same liver regions across all of the 7 volunteers. For the kidney, the selected landmarks were the caudal boundary (landmark \# 1) and the two extremities of the major calyx in the cranio-caudal direction (landmarks \# 2 and \# 3). In case not all of the landmarks would be visible in a single sequence, additional imaging would be performed on the volunteer, with the FOV focused on the missing landmark(s). Note that, in some cases, the breathing dynamics of the volunteer would change in between the sequences (i.e. becoming deeper or shallower). The landmarks were manually tracked on a time-frame of $\sim 8 \mathrm{~s}$ (which usually included 2-3 breathing cycles), and the coordinates of the each landmark were stored and used as a gold standard. The displacements in each liver and kidney dataset were then estimated on a pixel-by-pixel basis via the EVolution algorithm and the resulting coordinates of the same landmarks were compared against the manually established gold standard. The comparison was performed both visually, by plotting the estimated and the manually determined trajectory for the landmarks, and by the evaluating the target registration error (TRE) (Maurer et al. 1997) defined as:

$$
\operatorname{TRE}[\mathrm{mm}]=\sqrt{\left(x_{I}+u\left(\vec{r}_{I}\right)-x_{J}\right)^{2}+\left(y_{I}+v\left(\vec{r}_{I}\right)-y_{J}\right)^{2}}
$$

where $\left(x_{I}, y_{I}\right)$ and $\left(x_{J}, y_{J}\right)$ are the manually determined landmark coordinates in the reference and the moving image, and $\left(u\left(\vec{r}_{I}\right), v\left(\vec{r}_{I}\right)\right)$ is the displacement estimated by the EVolution algorithm for that particular landmark.

2.2.2. Standoff echography study A system was developed at our institute that allows clinical HIFU interventions under MR and/or US guidance (see Fig. 1(a)). The system is a modified Philips Sonalleve abdominal HIFU platform (Philips Healthcare, Vantaa, Finland), which is integrated in a 1.5T Philips Achieva scanner (Philips Healthcare, Best, Netherlands). Therapeutic HIFU energy in this modified system is provided by a custom-built sparse spherical phased transducer (IMASONIC, Voray sur l'Ognon, France), which replaces the original spherical transducer and is described in detail here (Ramaekers et al. 2017). The custom-built transducer is composed of 256 elements, 
operates at a frequency of $1.3 \mathrm{MHz}$ and has an aperture diameter and radius of curvature of $16 \mathrm{~cm}$. Embedded in the therapeutic matrix of the transducer is a linear 128element single-crystal linear phased array, which operates at a frequency of $3.5 \mathrm{MHz}$ (see Fig. 1(b)). The imaging array provides a co-axial image of the focus area of the HIFU transducer at $16 \mathrm{~cm}$. Both the therapeutic array as well as the embedded USimaging array are passively and actively MR-compatible and thus allow full simultaneous operation with real-time MRI in absence of mutual interference. A passively RFshielded Verasonics beamformer (Kaczkowski \& Daigle 2011, Verasonics 2017), which can thus also be operated in the Faraday cage of the MR-scanner, is used over a shielded cable connector to drive the imaging probe. The beamformer is operated via a fiberoptical PCIe link from a computer located adjacent to the MR-scanner console outside the Faraday cage. Time synchronization between the MR-acquisition level and the Verasonics beamformer was achieved by the means of a dedicated TTL line between the two systems and a Cortex M3 microcontroller as an intermediary. A third TTLconnection from the microcontroller allowed additional time synchronization of the HIFU-generator. This setup allowed the following two configurations, which were used in the scope of this paper: 1) MRI as the master-clock and the US-imager and the HIFU system slaved (with independent delays) and 2) The microcontroller as the master-clock with both HIFU and US-imager slaved, while the MRI runs asynchronously.

One purpose of the standoff echography study was to analyze the tracking capabilities of the EVolution algorithm as a means for B-mode US-based motion compensation during hybrid MR-US-guided HIFU therapies. Two separate experiments were conducted in this regard: one on a gel phantom undergoing a known motion pattern and an in-vivo study on three healthy volunteers. A third independent experiment was carried-out on a gel phantom in order to ensure that HIFU ablations are possible with minimal interference between the HIFU therapeutic signal and the US imaging signal.

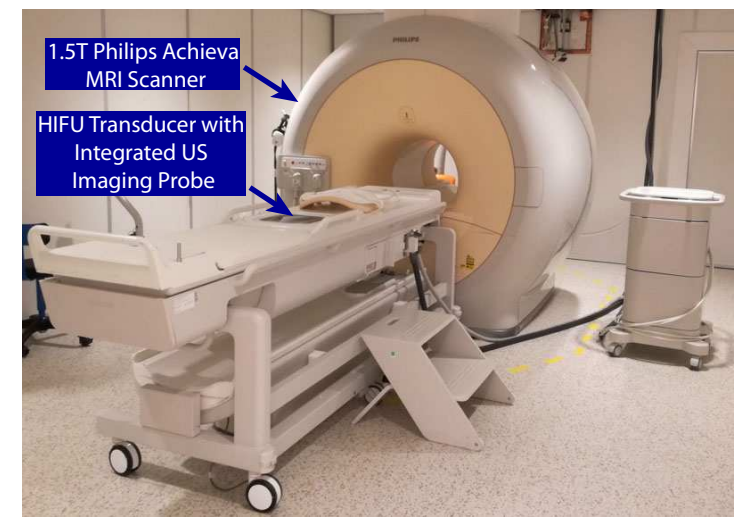

(a)

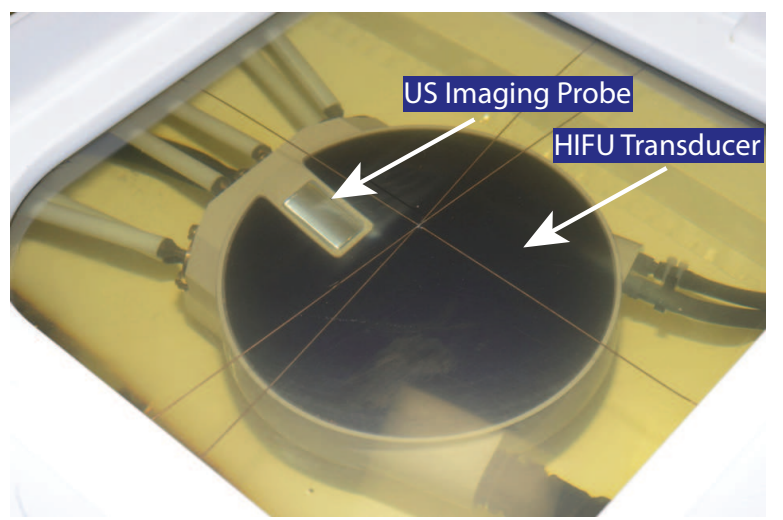

(b)

Figure 1: The custom-built HIFU system developed at our institute, which can provide MR and/or B-mode US guidance. (a): Overall setup. (b): The HIFU transducer together with the integrated US imaging probe. 
Ex-vivo standoff echography phantom experiment Since motion estimation algorithms typically rely on structural information in order to provide accurate displacements, a custom phantom was built for the current experiment. The phantom consisted in two perpendicular grid-like structures placed inside a cylindrical plastic casing, provided with a lid on one end and with an acoustic-transparent mylar membrane at the other. Prior to sealing the plastic case, the phantom was filled with an acoustically semi-transparent polymer gel. The phantom was then placed on a motorized platform, which allowed to move the US-phantom in a linear fashion over the acoustic window of the HIFU platform. The motor of the platform (USR60-E3T, Shinsei Corporation, Tokyo Japan) was controlled by an embedded microcontroller (ARM Cortex M3), which provided full closed-loop proportional-integral-derivative (PID) control over the linear motion pattern. In the scope of the experiment, a motion pattern corresponding to the average head-foot liver displacement of a free-breathing volunteer from previous studies (Zachiu et al. 2015) was loaded into the controller algorithm (duration $\sim 90 \mathrm{~s}$, with an original sampling density of $6 \mathrm{~Hz}$, up-sampled by sinc interpolation to $100 \mathrm{~Hz}$ ). Both the motion platform and the acoustic phantom were designed such that the HIFU transducer (and implicitly the US imager) had acoustic access to the phantom during the entire motion cycle.

Simultaneous MR and B-mode US imaging was performed on the moving phantom. The MR acquisition sequence was a multi-shot (4 shots per image) gradient recalled echo with an echo planar read-out, employing the following parameters: $\mathrm{TR}=45 \mathrm{~ms}$, TE $=$ $11 \mathrm{~ms}, \mathrm{FA}=25^{\circ}, \mathrm{BW}$ read $=1132 \mathrm{~Hz}$, image size $=176 \times 176$, pixel size $=2 \times 2 \times 7 \mathrm{~mm}^{3}$, acquiring a total of 500 images over a duration of $\sim 90 \mathrm{~s}$. Synchronously with the MRI, a B-mode US-image series was acquired using a 3-cycle pulse with a frequency of $3.5 \mathrm{MHz}$, image size $1024 \times 256$, a $0.25 \times 0.25 \mathrm{~mm}^{2}$ pixel size and at the same imaging rate as the MR sequence. The images were obtained using a spatial compounding over 6 angles (45 angle range) and synthetic aperture focusing, with the virtual source placed at a depth of $125 \mathrm{~mm}$ (Nikolov \& Jensen 2002, Frazier \& O'Brien 1998). Both the MR and the US images were saved on disk and processed offline. Timing-wise, for the synchronized MR-US acquisition, the US-imager is triggered with the acquisition of the first k-space segment of an MR-slice (which acquires the k-space center). After the trigger signal is received, the US-imager immediately acquires the 6 compounding angles, while the MRI acquires simultaneously the first k-space segment. The US-acquisition is completed with the first $\mathrm{k}$-space segment acquisition.

During the ex-vivo experiment, the motion pattern induced to the phantom by the motorized platform can be used as a gold standard. In comparison, for in-vivo scenarios, obtaining a reliable ground-truth in-vivo in a non-invasive manner can be a difficult task. For this reason, in anticipation to the in-vivo study described in the following section, the phantom experiment was used to validate both the EVolution algorithm applied on the US image series and the optical flow algorithm (Zachiu et al. 2015) applied on the simultaneously acquired MR images. The output provided by the optical flow algorithm on MR images will be used as a validation mechanism during the in-vivo study on the 
three healthy volunteers.

The phantom trajectory estimated in both the MR and the US image series was compared to the injected motion pattern. However, note that the MR and the US images were acquired in a different coordinate system. Thus, in order to ensure consistency between the displacements estimated on the two modalities, prior to registration, the MR and the US images were re-sampled on a common frame-of-reference. The axes of the new coordinate system were aligned with the axes of the MR images, whereby, the size of the the common reference frame was established at $256 \times 256$ with a $1 \times 1 \mathrm{~mm}^{2}$ pixel size. Moreover, prior to re-sampling, the far field of the US images was cropped-out, since for the current experiment it did not contain useful information. The US images also underwent a histogram equalization procedure. The average phantom displacements estimated from the two modalities were analyzed in relation to the injected motion pattern both in terms of followed trajectory and the pixel-wise error in flow endpoint (FEP) (Baker et al. 2011):

$$
\mathrm{FEP}[\mathrm{mm}]=\sqrt{\left(u_{\text {gold }}-u_{\text {est }}\right)^{2}+\left(v_{\text {gold }}-v_{\text {est }}\right)^{2}}
$$

where $\left(u_{\text {gold }}, v_{\text {gold }}\right)$ and $\left(u_{\text {est }}, v_{\text {est }}\right)$ are the gold standard and the estimated motion fields, respectively. For the current experiment, $u_{\text {gold }}$ is the displacement induced to the moving phantom, while $v_{\text {gold }}$ was set to 0 , since the moving platform only underwent a $1 \mathrm{D}$ translational motion. Thus, unlike the contact echography study, the gold standard here is a set of displacements, rather than a set of coordinates providing the location of a landmark over the US image series. By definition, the FEP relates two motion vector fields to one-another, reason for which, in this case, it was deemed to be a more suitable metric than the TRE.

In-vivo standoff echography study The current healthy volunteer study was conducted in agreement with the required standards and regulatory requirements. Ethical approval was provided by the Ethics Board of the University Medical Center Utrecht. Simultaneous MR and B-mode US imaging was carried-out on the abdomen of three healthy volunteers. The MR acquisition sequence was a multi-shot (9 shots per image) gradient recalled echo with echo planar readout employing the following parameters: $\mathrm{TR}=36 \mathrm{~ms}, \mathrm{TE}=11 \mathrm{~ms}, \mathrm{FA}=30^{\circ}, \mathrm{BW}_{\text {read }}=1131 \mathrm{~Hz}$, image size $176 \times 176$, voxel size $=2 \times 2 \times 7 \mathrm{~mm}^{3}$, acquiring a total of 300 images over a duration of $\sim 100 \mathrm{~s}$. The US acquisition sequence employed the same parameters and had the same timing as in the phantom experiment described in the previous section. The exact same preprocessing and processing steps as in the phantom experiment were carried-out on the data acquired on the healthy volunteers. However, as anticipated from the previous section, the displacements estimated by the EVolution algorithm on the US images were evaluated with respect to the ones estimated by the optical flow on the simultaneouslyacquired MR images (Zachiu et al. 2015). Motion analysis was conducted in a region of interest surrounding the lower boundary of liver segment \# 5, using the same motion quality evaluation criteria as in the phantom experiment. However, for the computation 
of the FEP, $\left(u_{\text {gold }}, v_{\text {gold }}\right)$ from Eq. 6 were replaced with the 2D displacements estimated by the optical flow algorithm on the MR series.

Interference avoidance scheme between the HIFU therapeutic signal and the B-mode US imaging signal For the system used in the standoff echography study, in case the HIFU transducer and the US imager operate simultaneously, the high amplitude of the the HIFU signal may completely saturate the low intensity electronics of the imaging system receiver. This will most likely lead to severe interference artifacts in the acquired B-mode US images, potentially affecting the tracking capabilities of the EVolution algorithm. In order to address this issue, previous studies propose implementing a synchronization scheme between the HIFU transducer and the US imager such that the HIFU beam is off during US-acquisitions (Vaezy et al. 2001). Another solution, which allows continuous operation of both the HIFU transducer and the US imager, is to filter the composite signal received by the US imager. In this sense, the HIFU therapeutic signal is attenuated to an extent that it has only minimal impact on the B-mode image quality (Jeong et al. 2009, Jeong et al. 2010, Jeong et al. 2012, Jeong et al. 2013, Takagi et al. 2016). In the scope of this study, the more technically simple solution of a timesynced acquisition was adopted, where US-imaging and HIFU are rapidly interleaved. HIFU-US synchronization was achieved using the setup described in the first paragraph of section 2.2.2.

In order to study the effectiveness of the proposed interference avoidance scheme, a HIFU heating experiment was conducted on a moving gel phantom. The phantom was placed on the same motorized platform as in the previous phantom experiment, however, the motion pattern induced here consisted in a simple sinusoidal displacement. A $300 \mathrm{~W}$ sonication was performed on the moving phantom, while continuously acquiring B-mode US images (frequency 20 images/s) over a duration of 55 s. MR-Thermometry (Rieke \& Pauly 2008) was simultaneously performed, in order to ensure that a lethal amount of thermal damage (Sapareto \& Dewey 1984) is achieved at the focus point. Both HIFU energy delivery and MR-Thermometry were gated using an MR pencil-beam navigator, however, the MR scanner and the US imager operated asynchronously. The quality of the B-mode US images was then evaluated both in the presence and in the absence of the proposed interference avoidance method.

\subsection{Configuration of algorithm input parameters}

The EVolution algorithm requires two parameters as input: the regularization parameter $\alpha$ and the patch size $\Gamma$ (see Eq. 1 and 2). An exhaustive optimization procedure was performed for both parameters. For both the contact and standoff echography studies, the value of $\alpha$ was varied between 0.1 and 1.0 with an increment of 0.05 , while the size of $\Gamma$ was varied between $3 \times 3$ and $21 \times 21$ with an increment of $2 \times 2$. The combination of $\alpha$ and $\Gamma$ which provided the minimum average TRE (for contact US) or FEP (for standoff US) was selected for use. For contact echography, the optimization procedure 
was carried-out per US sequence, with the average TRE including all three landmarks. For the standoff data, the average FEP was computed over the three volunteers.

The procedure described above also allowed the study of the algorithm's sensitivity to the two input parameters. This was achieved by evaluating the average TRE and FEP computed across all the contact and standoff sequences, respectively, for each combination of $\alpha$ and $\Gamma$.

\subsection{Implementation}

A computer unified device architecture (CUDA) implementation was performed for both the EVolution and the optical flow algorithms used to register the US and the MR images, respectively. The implementation was then executed on an nVidia Tesla K20 graphical processing unit (GPU).

\section{Results}

\subsection{Tracking quality evaluation for contact B-mode echography}

Fig. 2 showcases the tracking capabilities of the EVolution algorithm in one of the volunteers included in the contact echography study (see section 2.2.1). More precisely, Fig. 2(a) and 2(d) illustrate for the liver and kidney, the images used as positional reference during the tracking process. The red dashed lines in the two figures delineate the contour of the organs of interest, while the yellow numbered arrows indicate the location of the tracked landmarks. Fig. 2(b) and 2(c) plot the vertical and horizontal displacements of landmark \# 1 in the liver, resulting from both manual tracking (red curve) and the EVolution algorithm (blue curve). The same curves were plotted in Fig. 2(e) and 2(f) for landmark \# 2 in the kidney. A good similarity can be observed between manual tracking and the EVolution motion curves.

A quantitative evaluation of the algorithm's performance is provided in Table 1 for all 7 volunteers. The TRE between the manually determined and the estimated position of the tracked landmarks is reported for both the liver and kidney. The TRE was evaluated under the format mean \pm standard deviation both in the presence and in the absence of a registration procedure via the EVolution algorithm. The two statistics were computed on the TREs pooled from all time samples. The TRE in the absence of registration was evaluated by setting $u\left(\vec{r}_{I}\right)$ and $v\left(\vec{r}_{I}\right)$ in Eq. 5 to 0 , while keeping $\left(x_{I}, y_{I}\right)$ and $\left(x_{J}, y_{J}\right)$ unaltered. Since the latter were established as gold standard (thus it is assumed that their values are in correspondence with the true motion), by setting $u\left(\vec{r}_{I}\right)$ and $v\left(\vec{r}_{I}\right)$ equal to 0 , the TRE provides the tracking errors/misalignments that would occur in the absence of a registration procedure. When tracking is enabled, a considerable improvement is attained in all analyzed cases, with a mean and standard deviation of the registration errors under $1.6 \mathrm{~mm}$ for most of the landmarks. It is only in a few isolated instances in which the accuracy drops to $1.6-1.7 \mathrm{~mm}$. 


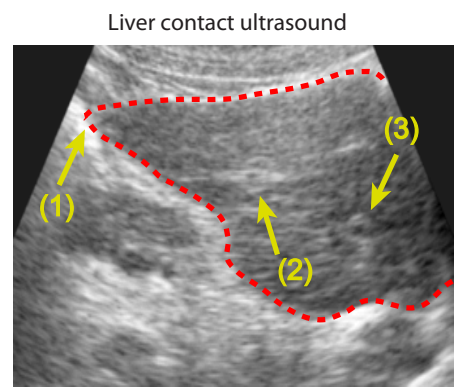

(a)

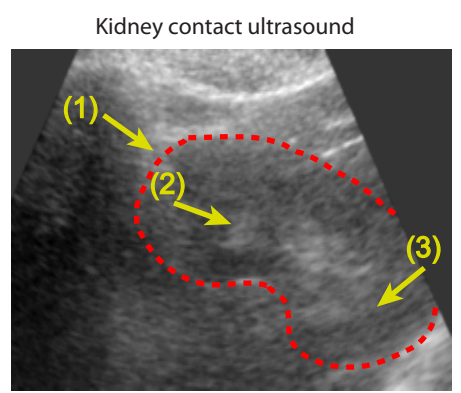

(d)

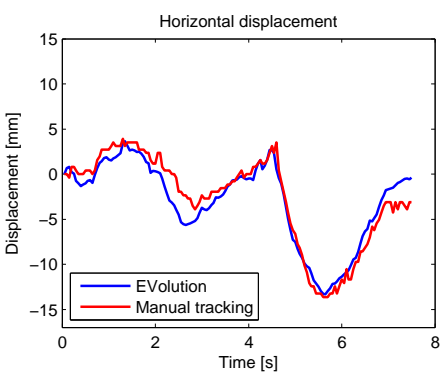

(b)

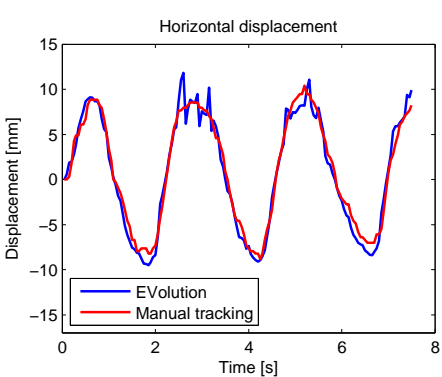

(e)

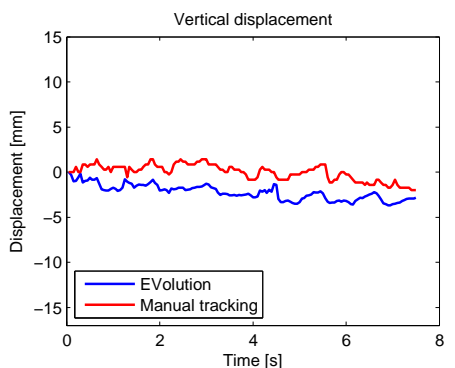

(c)

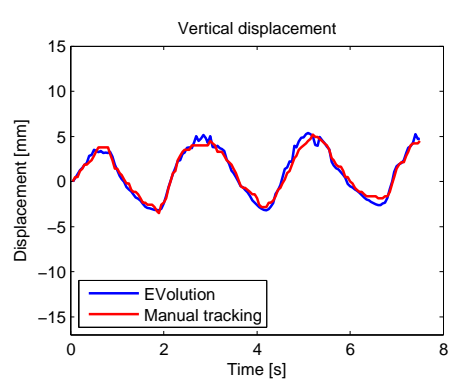

(f)

Figure 2: Tracking capabilities of the EVolution method for B-mode contact echography: (a), (d): US images used as positional reference for liver and kidney tracking, in one of the volunteers included in the contact echography studies. The red dashed line emphasizes the contour of the organs of interest while the yellow numbered arrows indicate the tracked landmarks. (b), (c): Horizontal and vertical displacements of landmark \# 1 in the liver. (e), (f): Horizontal and vertical displacements of landmark \# 2 in the kidney. The red curves are the manually determined displacements (used as a gold standard), while the blue curves correspond to the displacements estimated by the EVolution method.

\subsection{Tracking quality evaluation for standoff B-mode echography}

3.2.1. Phantom study The purpose of this experiment was to validate both the optical flow and the EVolution algorithm against a gold standard consisting in a known motion pattern induced to a phantom. Fig. 3illustrates the images used as positional reference during the phantom tracking study (detailed in section 2.2.2). Fig. 3(a) and 3(b) showcase the reference images for the MR and US series, in their original frame-ofreference. The yellow dashed line in Fig. 3(a) indicates the tracked structure. Fig. 3) (c) displays the two images in the same coordinate system. In order to show how the content of the two images relate to one-another, the US imaging plane was indicated on the MR image by a green dashed contour in Fig. 3(c). Also, the part where the two images overlap was replaced with the content of the US image, after its transformation in the new coordinate system.

Fig. 4 depicts the trajectory of the structure indicated by the yellow contour in Fig. 


\begin{tabular}{|c|c|c|c|c|c|}
\hline \multirow{3}{*}{$\begin{array}{l}\text { Volunteer } \\
\text { number }\end{array}$} & \multirow{3}{*}{$\begin{array}{c}\text { Landmark } \\
\text { number }\end{array}$} & \multicolumn{4}{|c|}{ Target registration error $[\mathrm{mm}]$} \\
\hline & & \multicolumn{2}{|c|}{ Liver } & \multicolumn{2}{|c|}{ Kidney } \\
\hline & & $\begin{array}{c}\text { Without } \\
\text { registration }\end{array}$ & $\begin{array}{c}\text { With } \\
\text { registration }\end{array}$ & $\begin{array}{c}\text { Without } \\
\text { registration }\end{array}$ & $\begin{array}{c}\text { With } \\
\text { registration }\end{array}$ \\
\hline \multirow{3}{*}{1} & $\# 1$ & $4.34 \pm 2.18$ & $1.35 \pm 0.88$ & $4.47 \pm 2.74$ & $1.58 \pm 0.76$ \\
\hline & $\# 2$ & $4.51 \pm 2.52$ & $1.09 \pm 1.00$ & $5.33 \pm 2.98$ & $1.54 \pm 0.65$ \\
\hline & $\# 3$ & $4.00 \pm 2.00$ & $1.77 \pm 1.07$ & $3.75 \pm 2.07$ & $1.23 \pm 0.64$ \\
\hline \multirow{3}{*}{2} & $\# 1$ & $4.28 \pm 3.94$ & $1.54 \pm 0.86$ & $5.97 \pm 3.17$ & $1.45 \pm 0.95$ \\
\hline & $\# 2$ & $5.90 \pm 2.82$ & $1.56 \pm 0.92$ & $6.01 \pm 2.91$ & $1.10 \pm 0.62$ \\
\hline & $\# 3$ & $6.31 \pm 3.27$ & $1.52 \pm 0.83$ & $5.37 \pm 2.39$ & $1.37 \pm 0.73$ \\
\hline \multirow{3}{*}{3} & $\# 1$ & $4.63 \pm 2.27$ & $1.61 \pm 0.88$ & $6.05 \pm 2.65$ & $1.52 \pm 0.65$ \\
\hline & $\# 2$ & $11.80 \pm 5.12$ & $1.47 \pm 1.06$ & $5.85 \pm 2.82$ & $0.90 \pm 0.43$ \\
\hline & $\# 3$ & $13.56 \pm 8.51$ & $1.43 \pm 0.60$ & $6.42 \pm 2.72$ & $1.49 \pm 1.05$ \\
\hline \multirow{3}{*}{4} & $\# 1$ & $3.61 \pm 2.00$ & $1.05 \pm 0.58$ & $2.86 \pm 1.20$ & $1.29 \pm 0.65$ \\
\hline & $\# 2$ & $3.55 \pm 2.87$ & $1.03 \pm 0.62$ & $4.95 \pm 3.99$ & $1.53 \pm 0.91$ \\
\hline & $\# 3$ & $4.11 \pm 2.63$ & $0.85 \pm 0.47$ & $5.23 \pm 3.17$ & $0.97 \pm 0.48$ \\
\hline \multirow{3}{*}{5} & $\# 1$ & $3.51 \pm 2.59$ & $1.19 \pm 0.80$ & $6.90 \pm 3.45$ & $1.50 \pm 0.98$ \\
\hline & $\# 2$ & $3.83 \pm 2.60$ & $1.77 \pm 0.97$ & $7.48 \pm 3.51$ & $1.25 \pm 0.73$ \\
\hline & $\# 3$ & $7.01 \pm 3.85$ & $1.42 \pm 1.05$ & $6.30 \pm 2.99$ & $1.24 \pm 0.53$ \\
\hline \multirow{3}{*}{6} & $\# 1$ & $5.41 \pm 3.06$ & $1.39 \pm 0.82$ & $5.58 \pm 3.32$ & $1.52 \pm 0.89$ \\
\hline & $\# 2$ & $6.76 \pm 3.55$ & $1.20 \pm 0.76$ & $3.67 \pm 2.02$ & $1.70 \pm 0.97$ \\
\hline & $\# 3$ & $6.34 \pm 2.94$ & $1.70 \pm 0.85$ & $4.96 \pm 3.08$ & $1.30 \pm 0.56$ \\
\hline \multirow{3}{*}{1} & $\# 1$ & $2.88 \pm 1.79$ & $1.16 \pm 0.55$ & $4.42 \pm 1.88$ & $1.57 \pm 0.67$ \\
\hline & $\# 2$ & $3.20 \pm 2.16$ & $1.53 \pm 0.66$ & $5.62 \pm 2.34$ & $1.45 \pm 1.04$ \\
\hline & $\# 3$ & $7.25 \pm 4.22$ & $1.10 \pm 0.60$ & $4.71 \pm 2.11$ & $1.35 \pm 0.66$ \\
\hline
\end{tabular}

Table 1: Accuracy and precision of the EVolution algorithm following the contact echography study on the 7 healthy volunteers. The table reports the TRE for the tracked landmarks in the liver and kidney, in the absence and in the presence of registration via the EVolution algorithm. Reporting is made under the format: mean \pm standard deviation.

3(a) during the first $\sim 60 \mathrm{~s}$ of the phantom study. The red curve corresponds to the known motion pattern induced to the phantom by the motorized platform, while the blue and the green curves are the average displacements estimated for the structure of interest by the optical flow and the EVolution algorithm, respectively, on the MR and the simultaneously acquired B-mode US image series. The trajectory is displayed only for the phantom's direction of travel, since the estimated magnitude of the perpendicular component was negligible. From a visual perspective, a good similarity between the red (used as a gold standard) and the other two curves can be observed. An evaluation of the FEP reveals a reduction of the alignment errors from $3.00 \mathrm{~mm} \pm 3.35 \mathrm{~mm}$ in the 


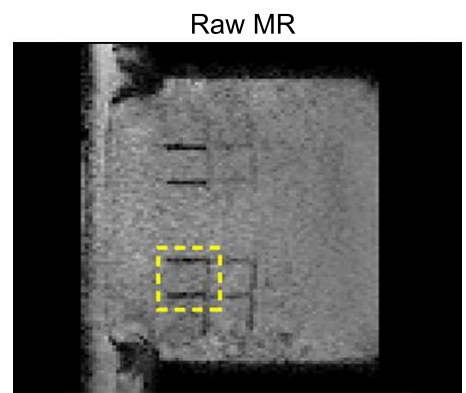

(a)

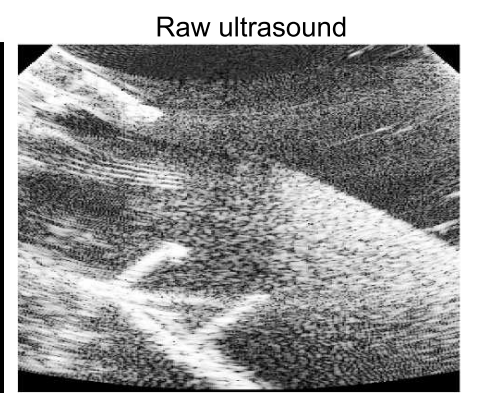

(b)

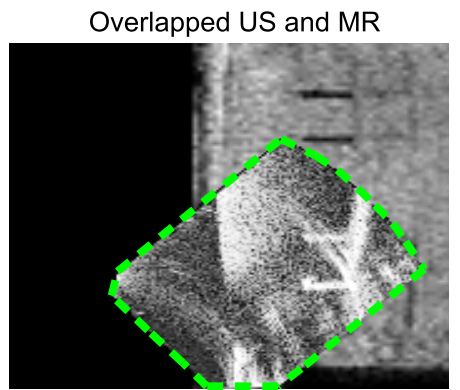

(c)

Figure 3: Example of data used during the phantom tracking study: (a), (b): Reference $\mathrm{MR}$ and its corresponding US image in their original coordinate system. The yellow dashed contour indicates the structure which was tracked during the experiment. (c): The MR and the US images in (a) and (b), projected onto a common reference frame. The green dashed contour delineates the US imaging plane in the illustrated composite image.

absence of tracking to $0.33 \mathrm{~mm} \pm 0.31 \mathrm{~mm}$ for the optical flow and $0.37 \mathrm{~mm} \pm 0.48 \mathrm{~mm}$ for the EVolution algorithm. The statistics on the registration errors were computed based on the pooled errors from all temporal sampling points.

\subsubsection{In-vivo healthy volunteer study The simultaneous MR-US healthy volunteer} study had the purpose of showcasing the performance of the EVolution tracking method for in-vivo standoff B-mode echography. Similar to Fig. 3. Fig. 5 displays the reference MR and US images acquired on one of the healthy volunteers, both in their original (Fig. 5(a) and 5(b)) and in a common coordinate system (Fig. 5(c)). The red dashed lines contour the visible part of the liver in the two images, while the blue lines delineate the abdominal wall. Again, in order to show how the content of the two images relate to one-another, the US imaging plane is indicated on the MR image in Fig. 5(c) by a green dashed line and the overlapping region was replaced with the transformed US image.

Fig. 6(a) illustrates, for volunteer \#3, the spatial distribution of the temporally averaged FEP between the motion vectors estimated by the optical flow and the EVolution algorithm, respectively, on the simultaneously acquired MR and US image series. The average FEP has been overlaid on the image used as reference during the registration of the standoff US image series. Note that the analysis was limited to a region of interest (ROI) (indicated by the dashed yellow contour in Fig. 6(a)) around the lower bound of liver segment \# 5. It can be observed that, for the majority of the pixels, the temporally averaged FEP rests below $2 \mathrm{~mm}$. Fig. 6(b) plots the temporal evolution of the spatially averaged displacements estimated for the liver of volunteer \#3. The average was calculated in the same ROI indicated by the yellow contour in Fig. 6(a). The analysis was conducted only for the head-foot component, since the displacement 


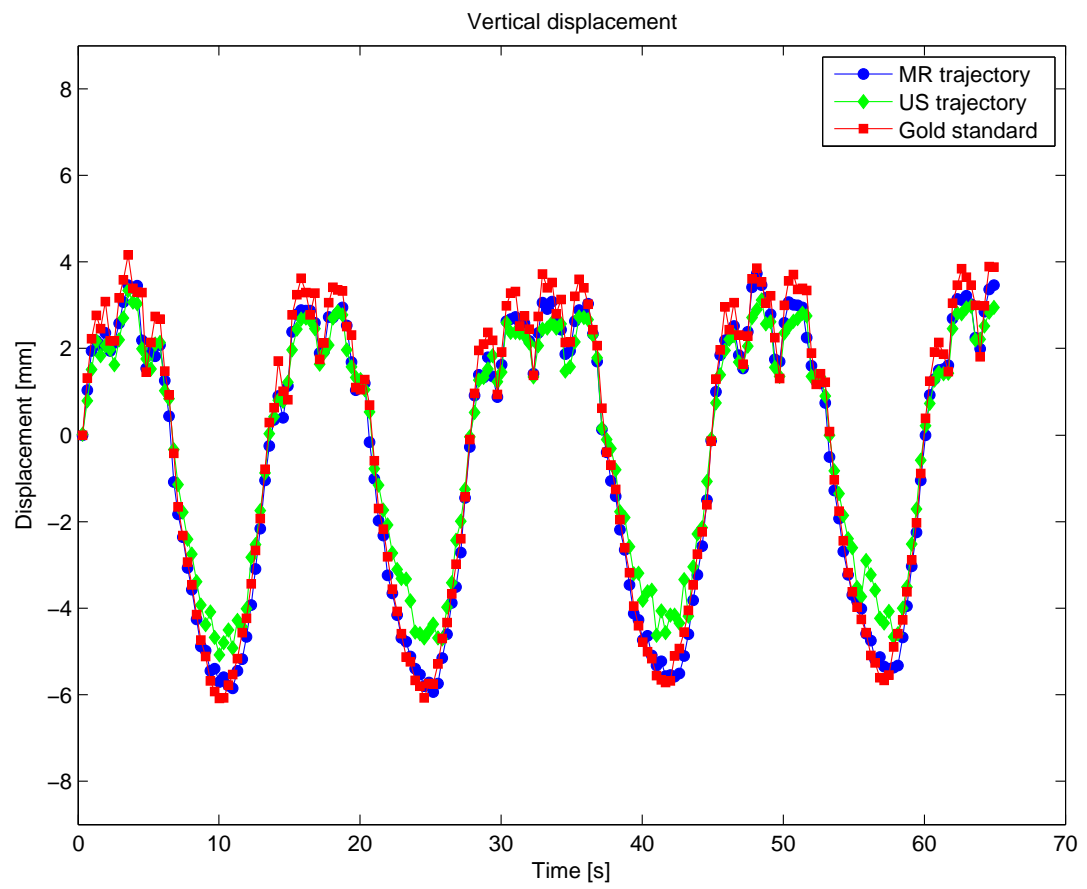

Figure 4: Performance evaluation of the optical flow and the EVolution algorithms following the phantom tracking experiment: The average spatial displacement in the direction of travel of the structure of interest during the first $\sim 60 \mathrm{~s}$ of the study. The blue curve corresponds to the displacements estimated by the optical flow algorithm on the MR-image series, while the green curve showcases the displacements estimated by the EVolution method on the simultanously acquired US images. The red curve illustrates the motion pattern induced to the phantom via the motorized platform.

in the anterior-posterior direction was negligible. The blue curve corresponds to the displacements estimated by the optical flow algorithm on the MR image series, which played the role of silver standard in the current experiment, while the green curve is the trajectory estimated by the EVolution method on the simultaneously acquired US image sequence.

Table 2 reports the FEP for all 3 volunteers, in the absence and in the presence of the EVolution tracking method. Since $\left(u_{\text {gold }}, v_{\text {gold }}\right)$ in Eq. 6] stand for the true motion of the tracked structure, the FEP in the absence of the EVolution algorithm was computed by setting $\left(u_{e s t}, v_{\text {est }}\right)$ to 0 . The values in Table 2 report the mean \pm standard deviation of the spatially averaged FEP in a region of interest covering the inferior part of the liver, with the statistics computed on the pooled values from all temporal sampling points. Improvements can be observed in all cases, with both the accuracy and precision of the proposed tracking method residing beneath $1.5 \mathrm{~mm}$. Noteworthy is also the inter-subject variability in tracking performance.

3.2.3. Efficiency of the proposed interference avoidance scheme Sections 3.2.1 and 3.2.2 report the achievable accuracy and precision of the EVolution algorithm for standoff US- 


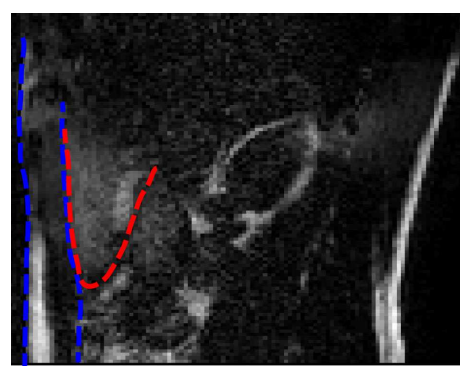

(a)

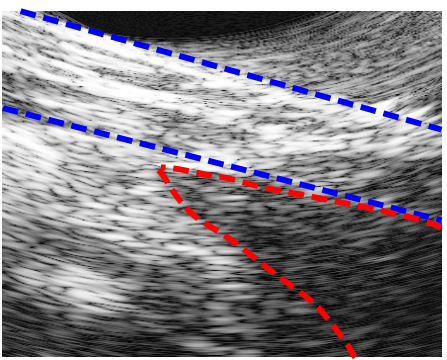

(b)

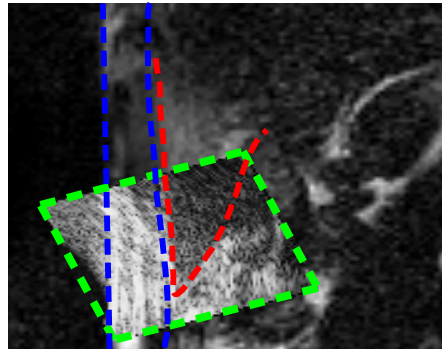

(c)

Figure 5: Example of data used for tracking during the in-vivo standoff echography study: (a), (b): The MR and its corresponding B-mode US image used as positional reference for volunteer $\# 3$, in their original coordinate system. In both (a) and (b) the red dashed line delineates the visible part of the liver, while the blue line delineates the abdominal wall. (c): The two images projected onto a common frame-of-reference. The green contour delineates the US imaging plane on the composite MR-US image. The red and the blue contours maintain the significance they had in (a) and (b).

\begin{tabular}{|c|c|c|}
\hline & \multicolumn{2}{|c|}{ Error in flow endpoint $[\mathrm{mm}]$} \\
\hline & Without registration & With registration \\
\hline Volunteer \#1 & $3.68 \pm 3.16$ & $1.3 \pm 1.2$ \\
\hline Volunteer \#2 & $2.55 \pm 2.87$ & $1.25 \pm 1.26$ \\
\hline Volunteer \#3 & $3.72 \pm 3.92$ & $0.8 \pm 1.01$ \\
\hline
\end{tabular}

Table 2: Accuracy and precision of the EVolution method following the standoff Bmode US study on the three healthy volunteers. Evaluation was performed for an ROI at the lower bound of liver segment \# 5, both in the presence (third column) and in the absence (second column) of the EVolution tracking method. The FEP in the absence of the EVolution algorithm was computed by setting $\left(u_{e s t}, v_{e s t}\right)$ to 0 in Eq. 6. The errors are reported under the format mean \pm standard deviation of the spatially averaged FEP pooled from all sampling time points.

based tracking. However, both the experiments were conducted with the HIFU beam off. As pointed-out in section 2.2.2, the HIFU therapeutic signal may oversaturate the signal received by the US imager and affect the quality of the US images and implicitly the performance of the tracking algorithm. Fig. 7 showcases such saturation effects in US images acquired during the experiment described in 2.2.2, paragraph 3. Fig. 7(a) illustrates one of the US images acquired while the HIFU transducer is off. Fig. [7(b) displays an US image acquired during a $50 \mathrm{~W}$ sonication in absence of an interference avoidance scheme. Note the strong interference artifacts that are present in the image, obscuring the imaged phantom. Fig. 7(c) displays an US image acquired during a 300 W sonication, with the proposed synchronization scheme between the HIFU transducer 


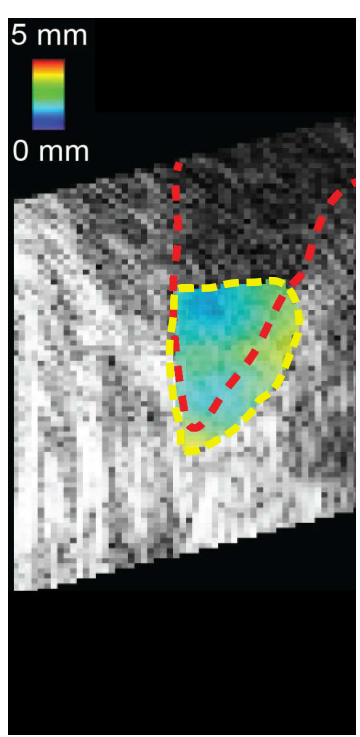

(a)

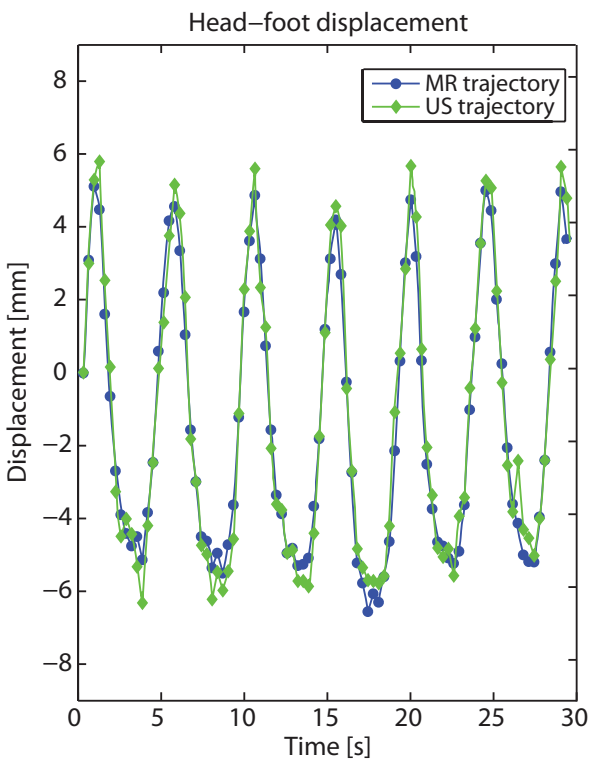

(b)

Figure 6: Performance evaluation of the EVolution method for in-vivo standoff B-mode echography. (a): Spatial distribution of the temporally averaged FEP calculated for volunteer \#3 and overlaid on the corresponding reference image of the standoff B-mode US image series. (b): The average liver displacement in the region-of-interest indicated by the yellow contour in (a), in the head-foot direction during the first $30 \mathrm{~s}$ of the study conducted on volunteer \#3. The blue curve illustrates the estimation provided by the optical flow algorithm on the MR image series (established as silver standard during the current experiment), while the green curve plots the one provided by the EVolution algorithm on the simultaneously acquired US image series.

and the US imager enabled. It can be observed that the saturation artifacts become visually unnoticeable.

Fig. 8(a) and 8 (b) showcase a coronal and a sagittal MR-Thermometry image acquired on the moving phantom during the HIFU heating experiment described in section 2.2.2, paragraph 3. The illustrated images were acquired at peak temperature, with the focal region magnified for better visibility. The white curve around the focal point indicates the region estimated to have received a lethal amount of thermal damage. This demonstrates that the proposed interference avoidance scheme does not hamper the system's ability for HIFU ablations. Moreover, no degradation in MR and US image quality was observed, despite the fact that the MR scanner and the US imager operated asynchronously during this experiment.

\subsection{Algorithm sensitivity to the choice of input parameters}

Fig. 9(a) and 9(b) illustrate the average TRE and FEP as a function of $\alpha$ and $\Gamma$ for the contact and standoff echography studies, respectively. For details on how these figures 


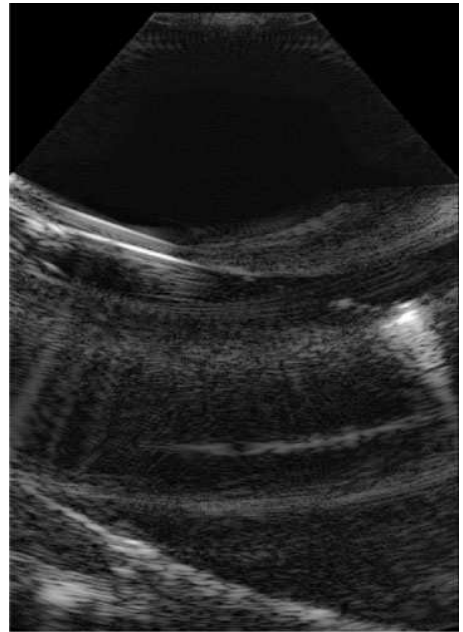

(a)

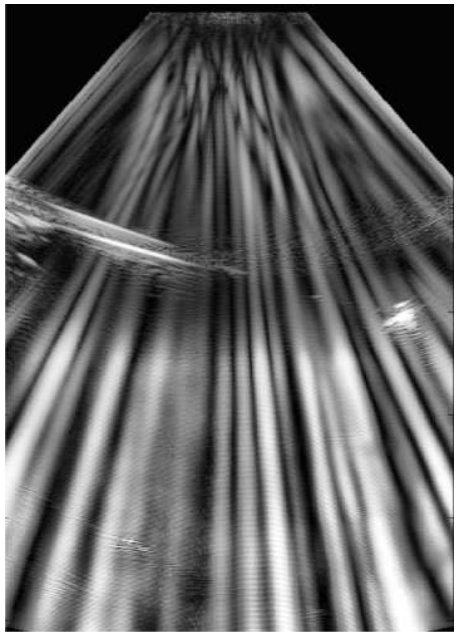

(b)

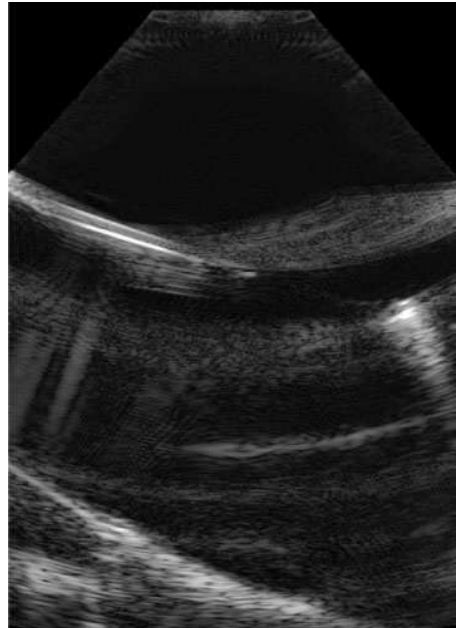

(c)

Figure 7: Efficiency of the proposed interference avoidance scheme between the HIFU therapeutic signal and the US imaging signal. (a) US image acquired while the HIFU transducer is off. (b): US image acquired during a $50 \mathrm{~W}$ HIFU sonication, without synchronization between the HIFU transducer and the US imager. (c): US image acquired during a $300 \mathrm{~W}$ HIFU sonication, with the interference avoidance scheme enabled.

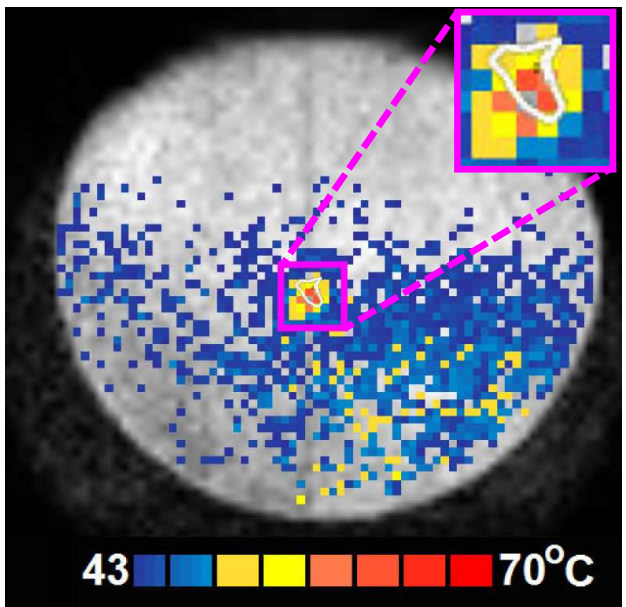

(a)

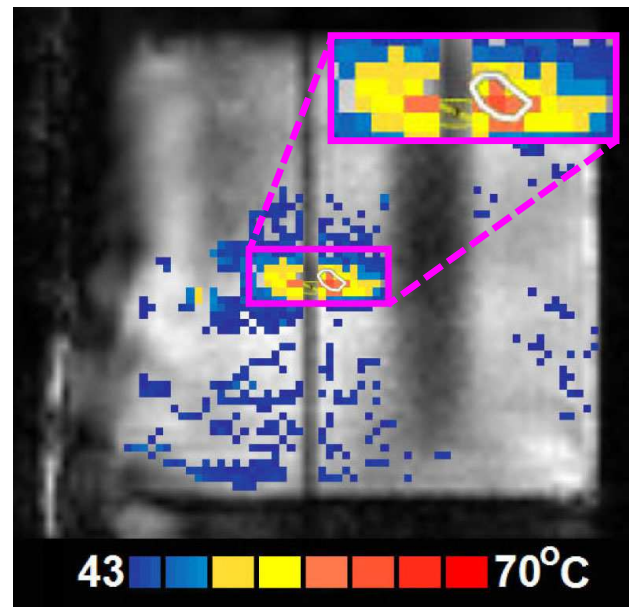

(b)

Figure 8: A (a): coronal (b): sagittal MR-Thermometry image acquired at peak temperature on the moving phantom during the heating experiment. The focal region was magnified for better visibility, with the white contour indicating the area estimated to have received a lethal amount of thermal damage.

were obtained, please see section 2.3. It can be observed that for contact echography, the maximum accuracy is attained for $(\alpha, \Gamma)=(0.3,15 \times 15)$, while for standoff echography the optimal combination is $(\alpha, \Gamma)=(0.35,3 \times 3)$. For both the contact and the standoff 
studies, the errors tend to be notably larger for small values of $\alpha$ and $\Gamma$, with a sudden drop at a value of $\sim(0.1,5 \times 5)-(0.15,7 \times 7)$. As the values of $\alpha$ and $\Gamma$ increase towards their maximum, the registration errors also show a slight tendency to increase.

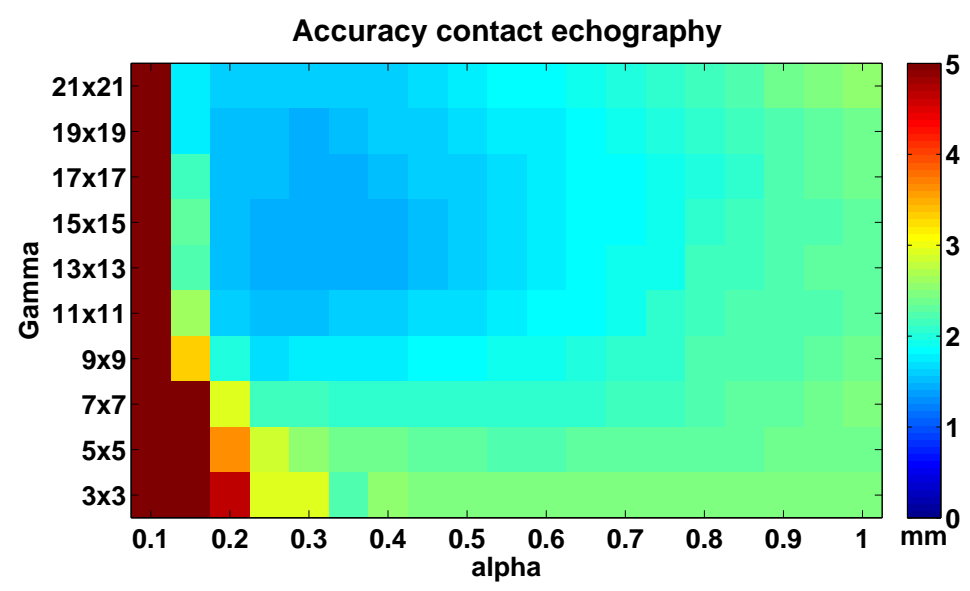

(a)

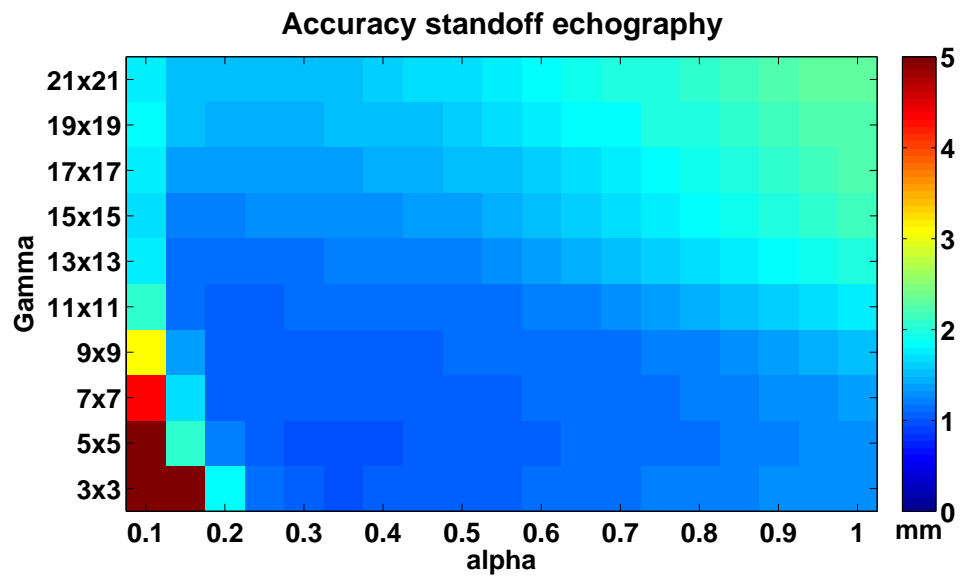

(b)

Figure 9: Sensitivity of the EVolution algorithm to input parameters $\alpha$ and $\Gamma$. Average (a): TRE and (b): FEP as a function of $\alpha$ and $\Gamma$ for the contact and standoff echography studies, respectively.

\subsection{Algorithm computational performance evaluation}

The GPU implementation of the EVolution algorithm converged on average in 50 ms. The convergence time was measured for images of size $256 \times 256$, which was the case for all data processed in the current study.

\section{Discussion}

The current study evaluates the real-time tracking capabilities of the recently proposed EVolution algorithm (Denis de Senneville et al. 2016) for both contact and standoff 
B-mode echography. The tracking method was chosen due to its intrinsic robustness to transient image structures (such as speckles in US images), fast convergence and reduced number of input parameters, while at the same time providing displacements on a pixel-by-pixel basis. According to the classification made in (De Luca et al. 2015b), the EVolution algorithm is a hybrid registration method. However, compared to existing hybrid methods for B-mode US registration, the EVolution algorithm has low computational demands, requires the configuration of a small number of input parameters, with no user interaction being necessary. Also, it does not require a learning step prior to its application, therefore its performance does not depend on the reproducibility of the estimated motion.

The evaluated tracking method was validated in three independent experiments, which were chosen as representative mock-ups of two clinical application scenarios: guidance of automated percutaneous interventions and HIFU therapies. The experiments encompassed an in-vivo contact echography study conducted separately on the liver and kidney of 7 healthy volunteers, a standoff echography study conducted on a phantom undergoing a known motion pattern and an in-vivo standoff echography experiment performed on the liver of three healthy volunteers. The low signal-to-noise (SNR) ratio, artifacts, poor contrast and orientation-dependent appearance of B-mode US images make tracking via gradient-based methods a challenging task (Jacob et al. 1999). It should also be noted that, in the scope of this study, with respect to the choice of the images, the employed equipment and acquisition schemes, no particular selection with respect to image quality has been made. Despite all this, the EVolution algorithm, which is gradient-based, provided in the majority of the analyzed cases, for both contact and standoff echography, motion estimates with an accuracy and precision higher than $1.6 \mathrm{~mm}$, which is on par with state-of-the-art algorithms dedicated to B-mode US image registration (De Luca et al. 2015a, De Luca et al. 2015b). However, while comparable, there is still a particular amount of inter-experiment and inter-subject variability in terms of tracking accuracy and precision. The cause to this is most likely variations in image quality (i.e. SNR, artifacts, etc.) between the different US image series and the US images themselves. Moreover, during the in-vivo studies, due to the limited acoustic window of the employed B-mode US imager, parts of the organs-of-interest would periodically enter and leave the field-of-view (FOV). In the scope of this paper, the tracked landmarks/ROIs were purposefully maintained in the FOV during the acquisition process. However, this aspect requires special attention when tracking is performed in a clinical setting, such that the region-of-interest does not leave the FOV. Also, note that the evaluation in the standoff echography study was limited to the lower bound of liver segment \# 5. The rather large distance between the US probe and the organ-of-interest led to a poor signal in regions towards the interior of the liver. Due to a lack of visible structures (such as organ boundaries and/or blood vessels), the algorithm performed poorly in these areas. Therefore, future work should should focus on improving the quality of the standoff US images acquired on the hybrid MR-US HIFU system.

One of the challenges that occurred during the validation of the proposed tracking 
method in the in-vivo scenarios was the choice of a reliable ground truth. For the in-vivo contact echography study the gold standard was determined by the means of manual tracking of identifiable anatomical landmarks. While a reliable approach, it is still sensitive to the observer's subjectivity, especially given the time-varying visibility of the structures in the US images over the respiratory cycle. For the standoff case, since a time-synchronized MR-image series was also acquired, a natural choice seemed the deformation fields estimated by the optical flow algorithm (Zachiu et al. 2015) on the MRimages. This was motivated by the fact that the optical flow algorithm was previously validated for MR-based motion tracking by several independent studies in both ex-vivo and in-vivo scenarios (Ostergaard et al. 2008, Castillo et al. 2009, Brock 2010, Kadoya et al. 2014). Furthermore, the good MR-based tracking capabilities of the optical flow algorithm was further confirmed in the current study during the phantom tracking experiment (see section 3.2.1). Another aspect that needs to be taken into consideration when evaluating the in-vivo tracking errors of the proposed method is that the imaging (and implicitly tracking) was performed in 2D. While the imaging planes were optimized such that they are in good correspondence with the principal axis of respiratory displacements, out-of-plane motion still remains a possibility. In case the latter becomes significant, its effects on the image content may be interpreted by the algorithm as inplane motion, leading to estimation errors. In such instances 3D US-tracking may be necessary (which has not been investigated in the scope of this study) or a method that includes through-plane motion detection capabilities could be employed, as described in (Ta et al. 2014).

The algorithm performance may also depend on the local validity of the smoothness assumption made by the second term of the functional in Eq. 1. For abdominal organs in particular, such a constraint is generally justified, since their composing tissues are incompressible and elastic. Thus, from a physical point-of-view, the displacement of the organs under discussion is indeed spatially smooth. Problematic are, however, the interfaces between the moving organs and the quasi-static abdominal/thoracic wall, where sliding/shearing motion may occur. Since this implies a local violation of the smoothness assumption, such areas are prone to motion estimation errors. More specifically, the displacements estimated for the mobile structures will get spatially propagated towards the static ones. Such a phenomenon was observed, for example, in the results associated to the in-vivo data displayed in Fig. 5. Due to the propagation of the displacements estimated for the liver, false motion was estimated for the quasi-static peritoneal wall. Area-wise, this effect extended $\sim 2-3$ pixels into the wall. A similar scenario can arise due to ghost image (aliasing) artifacts caused by acoustic multiple reflection. The latter can lead to some objects/structures appearing at several locations in the US image: once at their true spatial location and the rest, due to the additional reflections, at false locations, overlapping with the real structures that are situated at those particular sites. Such an effect is visible, for example, in Fig. 3(b), where a ghost of the HIFU transducer membrane partially overlaps with the tracked structure. The issue is that, in general, the ghost image of an object may or may not move relative to the real structure 
that it overlaps with. Thus, a smoothness assumption on the estimated displacements may not be valid. In the aforementioned example from Fig. 3(b), the reflection of the transducer membrane was static relative to the tracked structure. The constraint to provide a smooth deformation in the overlap region between the static membrane reflection and the moving structure, lead to a local decrease in the magnitude of the estimated motion vectors, especially for large displacements. This phenomenon may explain the systematic bias at the negative peaks of the green curve in Fig. 4. While in the scope of this study, neither sliding motion nor ghosting artifacts notably hampered the estimated displacements, as a source of mis-registration, they should be carefully monitored when tracking is performed via the EVolution method.

A feature that makes the EVolution algorithm an attractive tracking solution is that it requires the input of only two parameters: $\alpha$ and $\Gamma$ (see Eq. 11). Moreover, as shown in Fig. 9, registration errors remain close to their global minimum for a wide range of $\alpha$ and $\Gamma$ combinations. This is the case especially for the standoff experiments. Note, however, that the accuracy rapidly decreases as $\alpha$ and $\Gamma$ approach 0.1 and $3 \times 3$, respectively. On the other hand, the impact on accuracy is less important as $\alpha$ and $\Gamma$ increase towards their maximum. Therefore, in practice it is favorable to overestimate the values of $\alpha$ and $\Gamma$.

An important factor for motion tracking in general is the computational time required by the employed algorithm. In particular for interventional guidance of mini- or noninvasive procedures, the real-time performance of such an algorithm is paramount. With respect to this, two fundamental aspects have to be considered and differentiated: latency and throughput. In the scope of this study, latency is defined as the computational time required by the algorithm to register one pair of images. Registration methods that provide a dense deformation field between two images, are known to have high computational demands, making an implementation with real-time capabilities a challenging task (De Luca et al. 2015b). However, an implementation with short associated latencies for the EVolution algorithm is facilitated by the method's pixel-wise numerical scheme, which can be massively parallelized. As stated in section 3.4 this resulted in an average latency of $50 \mathrm{~ms}$ per registration, for images of size $256 \times 256$. Whether this is sufficient for real-time guidance depends on the particularities of the application. For example, for percutaneous interventions performed by a clinician using a handheld US device, the latency should reside below the average human reaction time. Since the latter is $\sim 200 \mathrm{~ms}$ (Woods et al. 2015), the evaluated tracking method fulfills this constraint with a considerable margin. It is, however, important to keep in mind that this latency may vary, depending on the extent of the deformation between the images, image size and the computational capabilities of the available hardware. Image throughput, on the other hand, refers to the number of images per unit time that a system is capable of processing. The implementation performed in the current study allowed for an image throughput of $\sim 20$ images per second, which is suitable for most state-of-the-art B-mode US imagers. The throughput can, however, be increased if necessary. For example, if two or more graphic cards of the same model as used in the presented work 
are included in the system, the image throughput can be scaled and adapted to imaging systems with high frame-rates (such as, for example, plane-wave US imagers (Montaldo et al. 2009)).

From a more practical side, the current work also proposes a scheme for addressing the interference between the US imaging and the HIFU signals. As illustrated in Fig. 17(b), if no interference avoidance measures are taken, a $50 \mathrm{~W}$ sonication may already lead to saturation artifacts that render the US images unusable for tracking. The proposed solution consists in a temporal multiplexing of the US imager and the HIFU transducer. As shown in Fig. 7(c), this removes the interference artifacts to an extent that they are no longer observable. A concern that arises with such a technique is that, since the HIFU transducer is turned off every time an US image is acquired, the thermal damage may be insufficient to have a therapeutic effect. This was, however, not the case in the current study since it was demonstrated that lethal thermal damage can still be achieved in the focal area (see Fig. 8). Nevertheless, such an approach, while efficient, it increases the time required to achieve this effect. This aspect can be addressed by employing more technically advanced solutions, which allow the continuous operation of the HIFU transducer and US imager. The principle behind such approaches involves encoding and/or filtering the composite signal received by the US imager, such that the HIFU therapeutic signal is strongly attenuated prior to image reconstruction (Jeong et al. 2009, Jeong et al. 2010, Jeong et al. 2012, Jeong et al. 2013, Takagi et al. 2016). Such techniques improve the duty cycle of the therapeutic energy delivery, leading to shorter sonication/therapy times compared to a temporal multiplexing solution. Nevertheless, in the scope of the current work, temporal synchronization between the two systems was deemed to be an acceptable solution, with more efficient approaches for interference avoidance being the topic of future studies.

The main focus of the current study was motion estimation. However, in particular for the hybrid MR-USg-HIFU system, a solution for motion compensation with respect to the estimated displacements would also be of interest. Motion compensation can be achieved, for example, by delivering the therapeutic energy in a gated manner. In effect, the HIFU transducer would be activated only when the targeted tissue is inside a pre-defined range of locations. Whether the tissue is situated in this range would be provided by the displacements estimated by the EVolution algorithm on the US images. Another solution for motion compensation would be electronic steering of the HIFU beam. In this sense, an approach similar to the one described by Ries et al (Ries et al. 2010) could be used, with steering being performed according to the motion vectors estimated by EVolution on the US image series.

\section{Conclusion}

A solution is proposed for target tracking in B-mode US sequences, capable of providing real-time guidance for US-guided percutaneous clinical interventions in 
mobile/deformable organs. The method is based on the recently proposed EVolution registration algorithm, a dynamic contour tracking method mathematically formulated in a variational setting. EVolution is a hybrid registration method which, as the current study demonstrates, showcases the advantages provided by both intensity-based and feature-based methods. It was also shown to be robust against their individual drawbacks. Similar to intensity-based registration methods, EVolution estimates a dense deformation between two images and does not require the selection/detection of image features. However, while intensity-based methods providing a dense deformation field usually imply high computational demands, in the current study it was shown that the EVolution algorithm has an image throughput and latency which are compatible with real-time B-mode US-guidance. Moreover, EVolution was shown to be capable of estimating rather large motion amplitudes of more than $10 \mathrm{~mm}$ and, by construction, is robust against transient structures, qualities which are specific to feature-based registration methods. However, since EVolution does not involve the selection/detection of a set of features, the registration process is not severely hampered by a low number of distinctive features in the images and/or the loss of features from one image to the next due to noise, image artifacts or through plane motion. In addition, even though B-mode US images are subject to low SNR, artifacts, poor contrast, and orientationdependent appearance, which renders the usage of gradient-based registration methods challenging, results have demonstrated that the EVolution algorithm is resilient against such effects, having on average an accuracy of $\sim 1.5 \mathrm{~mm}$ and submillimeter precision.

\section{Acknowledgments}

This work was supported by the Dutch Technology Foundation (STW) (project OnTrack no. 12813) and by the Seventh Framework Programme, grant no. 603028 (iPaCT). The authors also gratefully acknowledge the two anonymous referees whose remarks and suggestions have helped us greatly in improving the quality of the manuscript.

\section{References}

Auboiroux, V., Petrusca, L., Viallon, M., Goget, T., Becker, C. D. \& Salomir, R. (2012). Ultrasonography-based 2D motion-compensated HIFU sonication integrated with reference-free MR temperature monitoring: A feasibility study ex vivo, Phys Med Biol 57(10): N159-71.

Baker, S., Scharstein, D., Lewis, J. P., Roth, S., Black, M. J. \& Szeliski, R. (2011). A database and evaluation methodology for optical flow, International Journal of Computer Vision 92(1): 1-31.

Blana, A., Rogenhofer, S., Ganzer, R., Lunz, J. C., Schostak, M., Wieland, W. F. \& Walter, B. (2008). Eight years' experience with high-intensity focused ultrasonography for treatment of localized prostate cancer, Urology 72(6): 1329-1333.

Brock, K. (2010). Results of a multi-institution deformable registration accuracy study(MIDRAS), Int J Radiation Oncology Biol. Phys. 76(2): 583-596.

Castillo, R., Castillo, E., Guerra, R., Johnson, V. E., McPhail, T., Garg, A. \& Guerrero, T. (2009). A framework for evaluation of deformable image registration spatial accuracy using large landmark point sets, Phys. Med. Biol 54: 1849-1870. 
Chapman, A. \& ter Haar, G. (2007). Thermal ablation of uterine fibroids using MR-guided focused ultrasound - A truly non-invasive treatment modality, Eur Raiol 17(10): 2505-2511.

Chu, K. F. \& Dupuy, D. E. (2014). Thermal ablation of tumours: biological mechanisms and advances in therapy, Nat Rev Cancer 14(3): 199-208.

Cifor, A., Risser, L., Chung, D., Anderson, E. M. \& Schnabel, J. A. (2013). Hybrid feature-based diffeomorphic registration for tumor tracking in 2-d liver ultrasound images, IEEE Trans Med Imaging 32(9): 1647 - 1656.

De Luca, V., Benz, T., Konodo, S., König, L., Lübke, D., Rothlübbers, S., Somphone, O., Allaire, S., Lediju Bell, M. A., Chung, D. Y., Cifor, A., Grozea, C., Günther, M., Jenne, J., Kipshagen, T., Kowarschik, M., Navab, N., Rühaak, J., Schwaab, J. \& Tanner, C. (2015a). The 2014 liver ultrasound tracking benchmark, Phys Med Biol 60(14): 5571-5599.

De Luca, V., Székely, G. \& Tanner, C. (2015b). Estimation of large-scale organ motion in B-mode ultrasound image sequences: A survey, Ultrasound Med Biol 41(12): 3044-3062.

Denis de Senneville, B., Zachiu, C., Ries, M. \& Moonen, C. (2016). EVolution: an edge-based variational method for non-rigid multi-modal image registration, Phys Med Biol 61(20): 73777396.

Dice, L. R. (1945). Measures of the amount of ecologic association between species, Ecology 26(3): 297 $-302$.

Dogra, V. S. \& Saad, W. E. A. (2009). Ultrasound-Guided Procedures, 1 edn, Thieme, New York, NY, United States.

Frazier, C. H. \& O'Brien, W. R. (1998). Synthetic aperture techniques with a virtual source element, IEEE Trans Ultrason Ferroelectr Freq Control 45(1): 196 - 207.

Gupta, S. \& Madoff, D. C. (2007). Image-guided percutaneous needle biopsy in cancer diagnosis and staging, Tech Vasc Interv Radiol 10(2): 88-101.

Hofer, S., Oberholzer, C., Beck, S., Looser, C. \& Ludwig, C. (2008). Ultrasound-guided radiofrequency ablation (RFA) for inoperable gastrointestinal liver metastases, Ultraschall Med 29(4): 388-392.

Holbrook, A. B., Ghanouni, P., Santos, J. M., Dumoulin, C., Medan, Y. \& Pauly, K. B. (2014). Respiration based steering for high intensity focused ultrasound liver ablation, Magn Reson Med 71(2): 797-806.

Horn, B. \& Schunck, B. (1981). Determining optical flow, Artificial Intelligence 17: 185-203.

Huang, X., Dione, D. P., Compas, C. B., Papademetris, X., Lin, B. A., Bregasi, A., Sinusas, A. J., Staib, L. H. \& Duncan, J. S. (2014). Contour tracking in echocardiographic sequences via sparse representation and dictionary learning, Med Image Anal 18(2): 253 - 271.

Huttenlocher, D. P., Klanderman, G. A. \& Rucklidge, W. (1993). Comparing images using the hausdorff distance, IEEE Trans. Pattern Anal. Mach. Intell. 15(9): 850 - 863.

Jacob, G., Noble, J. A., Mulet-Parada, M. \& Blake, A. (1999). Evaluating a robust contour tracker on echocardiographic sequences, Med Image Analisys 3(1): 63-75.

Jeong, J. S., Cannata, J. M. \& Shung, K. K. (2010). Adaptive HIFU noise cancellation for simultaneous therapy and imaging using an integrated HIFU/imaging transducer, Phys Med Biol 55(7): 1889 $-1902$.

Jeong, J. S., Chang, J. H. \& Shung, K. K. (2009). Ultrasound transducer and system for real-time simultaneous therapy and diagnosis for noninvasive surgery of prostate tissue, IEEE Trans Ultrason Ferroelectr Freq Control 56(9): 1913 - 1922.

Jeong, J. S., Chang, J. H. \& Shung, K. K. (2012). Pulse compression technique for simultaneous HIFU surgery and ultrasonic imaging: A preliminary study, Ultrasonics 52(6): 730 - 739.

Jeong, J. S., Yoo, Y., Song, T. K. \& Chang, J. H. (2013). Real-time monitoring of HIFU treatment using pulse inversion, Phys Med Biol 58(15): 5333 - 5350.

Kaczkowski, P. J. \& Daigle, R. E. (2011). The Verasonics ultrasound system as a pedagigic tool in teaching wave propagation, scattering, beamforming, and signal processing concepts in physics and engineering, $J$ Acoust Soc Am 129(4): 2648.

Kadoya, N., Fujita, Y., Katsuta, Y., Dobashi, S., Takeda, K., Kishi, K., Kubozono, M., Umezawa, 
R., Sugawara, T., Matsushita, H. \& Jingu, K. (2014). Evaluation of various deformable image registration algorithms for thoracic images, J Radiat Res 55(1): 175-182.

Kass, M., Witkin, A. \& Terzopoulos, D. (1988). Snakes: Active contour models, Int J Comp Vis 1(4): $321-331$.

Kaye, D. R., Stoianovici, D. \& Han, M. (2014). Robotic ultrasound and needle guidance for prostate cancer management: Review of the contemporary literature, Curr Opin Urol 24(1): 75-80.

König, L., Kipshagen, T. \& Rühaak, J. (2014). A non-linear image registration scheme for real-time liver ultrasound tracking using normalized gradient fields, MICCAI Challenge on Liver Ultrasound tracking, Boston, USA.

Kovatcheva, R. D., Guglielmina, J.-N., Abeshera, M., Boulanger, L., Laurent, N. \& Poncelet, E. (2015b). Ultrasound-guided high-intensity focused ultrasound treatment of breast fibroadenoma-A multicenter experience, $J$ Ther Ultrasound $\mathbf{3}(1)$.

Kovatcheva, R. D., Vlahov, J. D., Stoinov, J. I. \& Zaletel, K. (2015a). Benign solid thyroid nodules: US-guided high-intensity focused ultrasound ablationinitial clinical outcomes, Radiology 276(2): 597-605.

Kovesi, P. (2000). Phase congruency: A low-level image invariant, Psychol Res 64(2): 136 - 148.

Kubota, Y., Matsumura, A., Fukahori, M., Minohara, S., Yasuda, S. \& Nagahashi, H. (2014). A new method for tracking organ motion on diagnostic ultrasound images, Med Phys 41(9): 092901.

Li, M., Kambhamettu, C. \& Stone, M. (2005). Automatic contour tracking in ultrasound images, Clin Linguist Phon 19(6 - 7): $545-554$.

Li, Y. Y., Sha, W. H., Zhou, Y. J. \& Nie, Y. Q. (2007). Short and long term efficacy of high intensity focused ultrasound therapy for advanced hepatocellular carcinoma, J Gastroenterol Hepatol 22(12): 2148-2154.

Liang, K., Rogers, A. J., Light, E. D., von Allmen, D. \& Smith, S. W. (2010). 3D ultrasound guidance of autonomous robotic breast biopsy: Feasibility study, Ultrasound Med Biol 36(1): 173-177.

Liberman, B., Gianfelice, D., Inbar, Y., Beck, A., Rabin, T., Shabshin, N., Chander, G., Hengst, S., Pfeffer, R., Chechick, A., Hanannel, A., Dogadkin, O. \& Catane, R. (2009). Pain palliation in patients with bone metastases using MR-guided focused ultrasound surgery: a multicenter study, Ann Surg Oncol 16(1): 140-146.

Mani, V. \& Arivazhagan, S. (2013). Survey of medical image registration, Journal of Biomedical Engineering and Technology 1(2): 8-25.

Maurer, C., Fitzpatrick, J., Wang, M., Galloway, R., Maciunas, R. \& Allen, G. (1997). Registration of head volume images using implantable fiducial markers, IEEE Transactions on Medical Imaging 16: $447-462$.

Montaldo, G., Tanter, M., Bercoff, J., Benech, N. \& Flink, M. (2009). Coherent plane-wave compounding for very high frame rate ultrasonography and transient elastography, IEEE Trans Ultrason Ferroelectr Freq Control 53(3): 489-506.

Mou, Y., Zhao, Q., Zhong, L., Chen, F. \& Jiang, T. (2016). Preliminary results of ultrasound-guided laser ablation for unresectable metastases to retroperitoneal and hepatic portal lymph nodes, World J Surg Oncol 14: 165.

Nikolov, S. \& Jensen, J. A. (2002). Virtual ultrasound sources in high-resolution ultrasound imaging, Medical Imaging: Ultrasonic Imaging and Signal Procesing, Vol. 4687, International Society for Optics and Photonics, pp. 395-405.

Okada, A., Murakami, T., Mikami, K., Onishi, H., Tanigawa, N., Marukawa, T. \& Nakamura, H. (2006). A case of hepatocellular carcinoma treated by MR-guided focused ultrasound ablation with respiratory gating, Magn Reson Med Sci 5(3): 167-171.

Ostergaard, N. K., Denis de Senneville, B., Elstrom, U. V., Tanderup, K. \& Sorensen, T. S. (2008). Acceleration and validation of optical flow based deformable registration for image-guided radiotherapy, Acta Oncol. 47(7): 1286 - 1293.

Petrusca, L., Cattin, P., De Luca, V., Preiswerk, F., Celicanin, Z., Viallon, M., Arnold, P., Santini, F., Terraz, S., Scheffler, K., Becker, C. D. \& Salomir, R. (2013). Hybrid ultrasound/magnetic 
resonance simultaneous acuisition and image fusion for motion monitoring in the upper abdomen, Invest Radiol 48(5): 333 - 340.

Preiswerk, F., De Luca, V., Arnold, P., Celicanin, Z., Petrusca, L., Tanner, C., Bieri, O., Salomir, R. \& Cattin, P. C. (2014). Model-guided respiratory organ motion prediction of the liver from 2D ultrasound, Med Image Anal 18(5): 740-751.

Ramaekers, P., de Greef, M., Berriet, R., Moonen, C. T. W. \& Ries, M. (2017). Evaluation of a novel therapeutic focused ultrasound transducer based on Fermat's spiral, Phys Med Biol 62(12): 5021-5045.

Rieke, V. \& Pauly, K. B. (2008). MR thermometry, J Magn Res Imaging 27(2): 376 - 390.

Ries, M., Denis de Senneville, B., Roujol, S., Berber, Y., Quesson, B. \& Moonen, C. (2010). Real-time 3D target tracking in MRI guided focused ultrasound ablations in moving tissues, Magnetic Resonance in Medicine 64(6): 1704-1712.

Sapareto, S. A. \& Dewey, W. C. (1984). Thermal dose determination in cancer therapy, Int J Radiat Oncol Biol Phys 10(6): 787 - 800.

Seo, J., Koizumi, N., Funamoto, T., Sugita, N., Yoshinaka, K., Nomiya, A., Homma, Y., Matsumoto, Y. \& Mitsuishi, M. (2011a). Visual servoing for a us-guided therapeutic HIFU system by coagulated lesion tracking: A phantom study, Int J Med Robot 7(2): $237-247$.

Seo, J., Koizumi, N., Funamoto, T., Sugita, N., Yoshinaka, K., Nomiya, A., Homma, Y., Matsumoto, Y. \& Mitsuishi, M. (2011b). Biplane US-guided real-time volumetric target pose estimation method for theragnostic HIFU system, Journal of Robotics and Mechatronics 23(3): 400-407.

Seo, J., Koizumi, N., Mithuishi, M. \& Sugita, N. (2016). Ultrasound image-based visual servoing for moving target ablation by high intensity focused ultrasound, Int $J$ Med Robot pp. e1793-n/a.

Ta, C. N., Eghtedari, M., Mattrey, R. F., Kono, Y. \& Kummel, A. C. (2014). 2-tier in-plane motion correction and out-of-plane motion filtering for contrast-enhanced ultrasound, Invest Radiol 49(11): 707-719.

Takagi, R., Jimbo, H., Iwasaki, R., Tomiyasu, K., Yoshizawa, S. \& Umemura, S.-I. (2016). Feasibility of real-time treatment feedback using novel filter for eliminating therapeutic ultrasound noise with high-speed ultrasonic imaging in ultrasound-guided high-intensity focused ultrasound treatment, Jap J App Phys 55(7S1): 07KC10: $1-8$.

Thirion, J. P. (1998). Image matching as a diffusion process: An analogy with Maxwell's demons, Med Image Anal 2(3): 243 - 260.

Vaezy, S., Shi, X., Martin, R. W., Chi, E., Nelson, P. I., Bailey, M. R. \& Crum, L. A. (2001). Realtime visualization of high-intensity focused ultrasound treatment using ultrasound imaging, Ultrasound Med Biol 27(1): 33 - 42 .

Verasonics (2017). The vantage research ultrasound systems. URL http://verasonics.com/vantage-systems/

Wang, Y., Georgescu, B., Comaniciu, D. \& Houle, H. (2010). Learning-based 3D myocardial motion flow estimation using high frame rate volumetric ultrasound data, Int Symp Biomed Imaging, IEEE, Piscataway, NJ, USA, pp. $1097-1100$.

Weickert, J., Bruhn, A., Papenberg, N. \& Brox, T. (2003). Variational optic flow computation: From continuous models to algorithms, International Workshop on Computer Vision and Image Analysis (ed. L. Alvarez), IWCVIA03, Las Palmas de Gran Canaria.

Woo, J., Hong, B. W., Hu, C. H., Shung, K. K., Jay Kuo, C. C. \& Slomka, P. J. (2009). Non-rigid ultrasound image registration based on intensity and local phase information, J Sign Process Sys 54(1): $33-43$.

Woods, D. L., Wyma, J. M., Yund, E. W., Herron, T. J. \& Reed, B. (2015). Factors influencing the latency of simple reaction time, Front Hum Neurosci 9: 131.

Zaaroor, M., Sinai, A., Goldsher, D., Eran, A., Nassar, M. \& Schlesinger, I. (2017). Magnetic resonanceguided focused ultrasound thalamotomy for tremor: a report of 30 parkinsons disease and essential tremor cases, J Neurosurg 24: 1-9.

Zachiu, C., Papadakis, N., Ries, M., Moonen, C. T. W. \& Denis de Senneville, B. (2015). An 
improved optical flow tracking technique for real-time MR-guided beam therapies in moving organs, Physics in Medicine and Biology 60(23): 9003.

Zhang, L. \& Wang, Z. B. (2010). High-intensity focused ultrasound tumor ablation: review of ten years of clinical experience, Front Med China 4(3): 294-302. 